\title{
Higher-Genus Contributions to an Induced Supergravity Action
}

\author{
Simon Davis \\ Received: January 5, 2020. Revised: August 24, 2nd Revised: October 1,ட2020. \\ Accepted: October 6, 2020. Published: October 9, 2020.
}

\begin{abstract}
The higher-genus contributions to a supergravity action induced by the evaluation of the superstring path integral are considered. Three extra terms are found in the BRST transformation that would arise in the supergravity action. The supersymmetric BRST contour integral over the ideal boundary of an infinite-genus surface is found to be vanishing if the harmonic measure is zero. The effect of a conformal transformation is demonstrated to be consistent with the vanishing of the BRST superspace contour integral. Since the commutator of BRST and conformal Since the BRST symmetry and conformal transformations transofrmations is proportional to the BRST current in four-dimensional gravity, and the vacuum is invariant under both transformations, conditions on the worldsheet fields result from the dynamics in the embedding space. A connection between conformal models on the two-dimensional worldsheet and the quantum action of the gravitational theory in four dimensions is established through the ghost sector. The number of degrees of freedom in the gravity theory must be reduced to achieve this result. The introduction of new variables related to the embedding of two-dimensional surfaces in three-geometries and then fourmanifolds is then required to satisfy the condition of quantum consistency characteristic of conformal field theories.
\end{abstract}

Keyword -induced supergravity, superspace contour integrals, ideal boundary, embedding space.

\section{Introduction}

The weighting factor in the bosonic string path integral $e^{\chi}$ is equal to $e^{-\int_{\Sigma} d^{2} \xi \sqrt{ } h R}$, where $R$ is the curvature of the surface $\Sigma$. This action also represents two-dimensional gravity, which is a topological theory with no classical dynamical degrees of freedom. Conformal rescalings of the metric of the surface introduce a scalar field $\phi$ such that the string coupling is given by the expectation value $\left\langle e^{\phi}\right\rangle$. Then the sum over the genus includes a factor $\left\langle e^{\phi}\right\rangle^{\chi}$ and which is expectation value of $e^{-S}$, where $S$ is a dynamical two-dimensional action $\int d^{2} \xi \sqrt{ } h \phi R$.

A similar effect occurs in connection with the quantum anomaly that prevents the Weyl and diffeomorphism transformations from being symmetries of the bosonic string action. The cancellation of this anomaly requires the introduction of counterterms that comprise the WessZumino-Polyakov action. Since the counterterm is generated by a BRST variation of this action, it entire integral must be invariant under BRST and conformal transformations.

The Wess-Zumino-Polyakov action is knwon for the complex plane to be the integral $-{ }_{2}^{1} d^{2} z \mu \partial^{2} \ln \lambda$, where $\mu$

Research Foundation of Southern California, 8861 Villa La Jolla Drive \#13595, La Jolla, CA 92037, U.S.A. is the Beltrami differential and $\lambda$ is the conformal factor in the combination of Weyl rescaling and diffeomorphisms [1]. The complex plane is the covering space of the torus, and both the solution to Ward identities for the conformal symmetry may be verified. However, it is not a universal covering space for genus $g \geq 2$ and represents only an intermediate covering after the isometric disks of the generators of the uniformizing Schottky groups are removed. Consequently, integrals in the action constructed with $\operatorname{PSL}(2 ; \mathbb{C})$ transformations must be defined on the fundamental domain consisting of the set of ordinary points on the complex plane. Nevertheless, a formula for the anomaly may be given. These fields can be projected onto two-dimensional string worldsheets [2][3].

The generalization to the superstring action will induce a supergravity action with fermion fields. In addition to diffeomorphism invariance, the string theory will characterized by supersymmetry and a BRST symmetry [4]. The BRST operator will occur in the formulation of the two-dimensional action, which is distinguished from the superstring effective action in the target space. There are higher-genus contributions to the supergravity action unless BRST invariance is preserved. The action of the BRST operator on the relevant terms in the action is zero if supersymmetric generalizations of the BRST contour integrals vanish. The integration over the ideal boundary could yield additional terms to the action. The breaking of any symmetries or the diminishing of the effect in the infinite-genus limit will be examined.

A fundamental result in string theory will be a theoretical foundation for the dynamics of matter and gravity in four dimensions. A Lagrangian with curvature terms is found to occur both in the string effective action and the induced supergravity action. The first is formulated in a higher critical dimension, and many new fields are introduced in the compactification to four dimensions. The second only requires an increase in the dimension of the model from two to four. There are many basic symmetries in these dimensions which are directly related to the geometry of Riemann surfaces and hyperbolic geometry. Therefore, much of the rigidity of the model, compatible with the finiteness of the quantum theory, can be preserved in this formalism.

It is demonstrated in $\S 2$ that there are three terms that could arise in the induced supergravity action on a Riemann surface. Two of the terms are given by differentials. These terms can be demonstrated to vanish over compact surfaces and surfaces with boundaries over which the contour integral vanishes, which is valid when 
the harmonic measure of the ideal boundary is zero. It is proven in $\S 3$ that this condition is equivalent on a Type I surface to the commutativity of the BRST and conformal transformations. The third integral equals zero because the superspace derivative and the BRST operator anticommute.

Therefore, the discussion of BRST transformations in the induced supergravity action on Riemann surfaces in string theory is complete. Since string theory and general relativity both describe the gravitational field in the embedding space, it is worthwhile verifying that several of the results in string theory also would be valid for a four-dimensional gravitational action. Such a result in established in $\S 3$, where it is found that the BRST and conformal symmetries of the ghost field action derived in the covariant quantization of the gravitational theory commute. Given this property, and the resemblance between the projection of the ghost Lagrangian to four dimensions to induced supergravity and $W_{3}$ gravity models, a basis for the transition from the worldsheet theory of strings to a gravitational theory in the embedding space theory now exists.

Two related results in gauge theories are the validity of the Adler-Bardeen theorem in establishing the nonrenormalization of anomalies, and the commutativity of conformal and BRST transformations. The absence of a rigorous proof of the supersymmetric generalization of the Adler-Bardeen theorem for a super-Yang-Mills theory has been related to noncommutativityof superconformal and BRST transformations [5]. One corollary is the absence of a conclusive demonstration of the gauge independence of the anomaly. Similarly, invariance of an anomaly under BRST transformations will not be evident.

For the induced supergravity action of the Polyakov string, the entire anomaly is constructed. The generalizetion to the superstring action will induce a supergravity action with fermion fields. In addition to diffeomorphism invariance, it follows from the previous discussion that supersymmetry transformations can cause the anomaly not to be preserved under BRST transformations, and a solution to the superconformal Ward identities is required. Consequently, the BRST transformations of all of the terms must be examined.

The action for general relativity in four dimensions can be quantized with the addition of a Faddeev-Popov Lagrangian and a gauge-fixing term. The BRST symmetry of the Faddeev-Popov action is supplemented by a conformal symmetry. Furthermore, the commutator of the BRST and conformal charges is found to be proportional to the BRST charge. Therefore, the vacuum can be defined such that it belongs to the kernel of both charges simultaneously. The commutator also would vanish upon evaluation in this physical state. Independence of the vacuum expectation value of the anomaly respect to BRST transformations then follows.

The projection of the four-dimensional Faddeev-Popov Lagrangian to two dimensions does not yield the bosonic string ghost action. The connection will be investigated further by considering a scalar form of the theory of gravity. In $\S 4$, it is recalled that equations for spin-two fields in the weak-field limit of general relativity yield a scalar differential operator that has a Green function decreasing with the inverse square of the distance and a momentum-space propagator which varies as the inverse square of the momentum. Therefore, the propagator and the quantum theory in its scalar form resemblescalar electrodynamics. Removing the field strength term, and using the metric connection to define an additional term in the covariant derivative, the symmetry of the Lagrangian with a local phase factor would be reduced to a global U(1) invariance under a gauge fixing condition. The projection of the ghost action of this model to a Riemann surface is found to include the ghost action of bosonic string theory. Ghosts representa complement to the space of physical states. Nevertheless, this result is indicative of a projection of quantum states in a scalar form of gravity in four dimensions to string states on two-dimensional surfaces.

The ghost sector complements the physical sector of the four-dimensional theory. Therefore, a further relation between the symmetries is necessary to establish a connection between the two-dimensional theory and a complete model of the dynamics in four dimensions, which is known not to be characterized macroscopically by conformal invariance. Two methods may be developed at this stage. First, the conformal gravity and supergravity Lagrangians may be included to extend the conformal symmetry from two to four dimensions. There are some problems of quantum consistency of the actions, together with relevance to gravitational phenonemona at large scales in space-time. Secondly, the escalation of the dimension from two to four dimensions follows a standard method. The two-dimensional rational conformal field theories are the dimensionally reduced Chern-Simons actions in three-dimensions. The ChernSimons action with gauge group $\operatorname{ISO}(2,1)$ is equivalent to three-dimensional gravity, which is a topological model. Consequently, embedding coordinates must be introduced to produce a four-dimensional action. These coordinates would describe the fibres of the tangent and normal bundles. The analogue of the Liouville action in string theory to describe hermitian structures in vector bundles and the dependence of the metric on the conformal scale factor is the Donaldson action, which may be given in both in two and four dimensions. The variational equation of the four-dimensional hermitian Einstein-Yang Mills integral is solved by anti-self dual connections. It may be recalled also that the Chern-Simons action on a threedimensional space $\Sigma$ is equal to the topological integral $\int_{M} \operatorname{Tr}(F \wedge * F)$, equal to the Yang-Mills action for a selfdual connection, on a manifold $M$ with boundary $\partial M=$ $\Sigma$, which exists always for oriented, closed, smooth threemanifolds. Therefore, the generalization of the worldsheet model to four dimensions, aligned with the quantum consistency of two-dimensional theories with conformal symmetry, may be developed through the quantization 
of these theories.

\section{The Vanishing of BRST Superspace Contour Integrals}

The classical Weyl and diffeomorphism invariance of the bosonic string does not remain at the quantum level since there is an anomaly described by a Wess-ZuminoPolyakov action. Given that there is a transformation of the coordiantes $(z, \bar{z})$ on a Riemann surface to $(Z, \bar{Z})$, where

$$
\begin{aligned}
& d Z=\lambda[d z+\mu d \bar{z}] \\
& d \bar{Z}=\bar{\lambda}[\bar{\mu} d z+d \bar{z}],
\end{aligned}
$$

with $\lambda=\partial Z$ being a conformal factor and $\mu=\frac{\bar{\partial} Z}{\partial Z}$ being the Beltrami coefficient. Calculations at leading order in the string perturbation series begin with surfaces of genus zero or the sphere. The evaluation of scattering matrix elements, such as the Kobe-Nielsen amplitude, does not require an additional moduli space integral, since the complex dimension of moduli space equals 0 for $g=0$, 1 when $g=1$ and $3 g-3$ if $g \geq 2$. Nevertheles, the above coordinate transformations exists on the complex plane the moduli space of metrics on the sphere is zero-dimensional. The removal of one point from the sphere produces a manifold that can be stereographically projected to the finite complex plane, which produces the torus upon identification of sides in a lattice. Therefore, the moduli space also would be one-dimensional, with the parameter being $\mu$. Therefore, the Wess-Zumino-Polyakov effective action must be regarded as an integral formulated over the moduli space at genus one, with the complex plane as the covering space. It is then defined [1] to be

$$
\left.\begin{array}{rl}
\Gamma_{W Z P}(\mu) & =-\frac{1}{2} \int_{\mathbb{C}} d^{2} z \mu \partial^{2} \ln \lambda=-\frac{1}{2} \int d^{2} z \frac{\bar{\partial} Z}{\partial Z} \partial^{2} \ln \partial Z \\
& =-\frac{1}{2} \int_{\mathbb{C}} d^{2} z\left\{\partial\left[\frac{\bar{\partial} Z}{\partial Z} \partial \ln \partial Z\right]-\partial\left(\frac{\bar{\partial} Z}{\partial Z}\right)\right. \\
\partial \ln \partial Z\} & =-\frac{1}{2} \int_{|z|=\infty} d \bar{z} \frac{\bar{\partial} Z}{\partial Z} \partial \ln \partial Z+\frac{1}{2} \int_{\mathbb{Z}} d^{2} z \partial\left(\frac{\bar{\partial} Z}{\partial Z}\right) \\
\partial \ln \partial Z
\end{array}\right\}
$$

such that it can be generalized to higher genus, even though there is no quotient by a lattice group. The first integral vanishes if $\mu(z, \bar{z})$ vanishes as $|z| \rightarrow \infty$ or the coordinates $(Z, \bar{Z})$ and $(z, \bar{z})$ coincide at infinity within a scaling factor. The second integral is

$$
\begin{array}{r}
\Gamma_{W Z P}(\mu)=\frac{1}{2} \int_{\mathbb{C}} d^{2} z\left(\frac{\partial \bar{\partial} Z}{\partial Z}-\frac{\partial^{2} Z}{\partial Z \bar{\partial} Z}\right) \frac{\partial^{2} Z}{\partial Z} \\
=\frac{1}{2} \int_{\mathbb{C}}\left[\left(\partial^{2} Z\right) \frac{\partial \bar{\partial} Z}{(\partial Z)^{2}}-\frac{\left(\partial^{2} Z\right)^{2}(\bar{\partial} Z)}{(\partial Z)^{3}}\right]
\end{array}
$$

Given the following transformations

$$
\begin{aligned}
s Z & =\lambda c \\
s \lambda & =\partial(\lambda c) \\
s \mu & =(\bar{\partial}-\mu \partial+\partial \mu) c \\
s c & =c \partial c,
\end{aligned}
$$

the variation of the effective action is

$s \Gamma_{W Z P}=-\frac{1}{2} \int_{\mathbb{C}} d^{2} z(s \mu) \partial^{2} \ln \lambda-\frac{1}{2} \int_{\mathbb{C}} d^{2} z \mu\left(s \partial^{2} \ln \lambda\right)$.

The BRST operator may be brought through the $\partial^{2}$ to give $\partial^{2} s(\ln \lambda)$ since $s Z=\lambda c$ and $s(\partial Z)=\partial(\lambda c)$. Then

$$
\begin{array}{r}
s \Gamma_{W Z P}=-\frac{1}{2} \int_{\mathbb{C}} d^{2} z(\bar{\partial}-\mu \partial-\partial \mu) c \partial^{2} \ln \lambda-\frac{1}{2} \int_{\mathbb{C}} d^{2} z \mu \partial^{2} \\
\frac{\partial(\lambda c)}{\lambda}=\frac{1}{2} \int_{\mathbb{C}} d^{2} z c(\bar{\partial}-\mu \partial) \partial^{2} \ln \lambda-\frac{1}{2} \int_{\mathbb{C}} d^{2} z \partial \mu c \partial^{2} \ln \lambda \\
-\frac{1}{2} \int_{\mathbb{C}} d^{2} z \mu\left(\partial^{3} c+\partial^{2}\left(c \frac{\partial \lambda}{\lambda}\right)\right) \\
=\frac{1}{2} \int_{\mathbb{C}} d^{2} z c\left[\partial^{2}\left(\frac{1}{\lambda}(\partial \mu \lambda)\right)-\partial^{2} \mu \partial \ln \lambda-\partial \mu \partial^{2} \ln \lambda\right] \\
-\frac{1}{2} \int_{\mathbb{C}} d^{2} z \mu\left(\partial^{3} c+\partial^{2}\left(c \frac{\partial \lambda}{\lambda}\right)\right) \\
\int_{\mathbb{C}} d^{2} z c\left(\partial^{2} \mu \partial \ln \lambda+\partial \mu \partial^{2} \ln \lambda\right)+\frac{1}{2} \int_{\mathbb{C}} d^{2} z c \partial^{2} z c \partial^{2} \mu \frac{\partial \lambda}{\lambda} \\
=\int_{\mathbb{C}} d^{2} z c \partial^{3} \mu-\frac{1}{2} \int_{\mathbb{C}} d^{2} z c(\partial \mu)\left(\partial^{2} \ln \lambda\right)
\end{array}
$$

The first integral is identified with $\int_{\mathbb{C}} d^{2} z \mathcal{A}(z ; \mu)$ which is the holomorphic component of the diffeomorphism anomaly.

The integrals in superspace require the basis elements of the cotangent space to the super-Riemann surface in the coordinates $(Z, \bar{Z}, \Theta, \bar{\Theta})$ with reference to the system $e^{z}=d z+\theta d \theta, e^{\theta}=d \theta$,

$$
\begin{aligned}
e^{Z} & =\left[e^{z}+e^{\bar{a}} H_{\bar{z}}^{z}+e^{\theta} H_{\theta}^{z}+e^{\bar{\theta}} H_{\bar{\theta}}^{z}\right] \Lambda \\
e^{\Theta} & =\left[e^{z}+e^{\bar{z}} H_{\bar{z}}^{z}+e^{\theta} H_{\bar{\theta}}^{z}+e^{\bar{\theta}} H_{\bar{\theta}}^{z}\right] z \\
& +\left[e^{\theta} H_{\theta}^{\theta}+e^{\bar{z}} H_{\bar{z}}^{\theta}+e^{\bar{\theta}} H_{\bar{\theta}}^{\theta}\right] \sqrt{\Lambda} .
\end{aligned}
$$

with $\Lambda=\partial Z+\Theta \partial \Theta$ and $\tau=\partial \Theta$. It follows from the structure equations [1], de $e^{Z}+e^{\Theta} \wedge e^{\Theta}=0$, de $e^{\Theta}=0$, that

$$
\begin{aligned}
\tau & =\frac{1}{\left(H_{\theta}^{\theta}\right)^{2}}\left(D-H_{\theta}^{z} \partial\right)\left(H_{\theta}^{\theta} \sqrt{\Lambda}\right) \\
\tilde{\bar{D}} \ln \Lambda & =\partial H_{\bar{\theta}}^{z}-\frac{H_{\bar{\theta}}^{\theta}}{H_{\theta}^{\theta}} \partial H_{\theta}^{z} .
\end{aligned}
$$

Given that there is a transition of coordinates from $(z, \bar{z})$ to $(\tilde{z}, \tilde{\bar{z}})$ to $(Z, \bar{Z})$, where $d \tilde{z}=\lambda(d z+\mu d \bar{z}), d \tilde{\bar{z}}=d \bar{z}$, $\tilde{\partial}=\lambda^{-1} \partial, \tilde{\bar{\partial}}=\bar{\partial}-\mu \partial, \tilde{D}=\partial_{\tilde{\theta}}+\tilde{\theta} \partial_{\tilde{z}}, \tilde{\bar{D}}=\partial_{\tilde{\theta}}+\tilde{\bar{\theta}} \partial_{\tilde{z}}$ and $\tilde{\bar{\partial}} \ln \lambda=\frac{1}{\lambda} \tilde{\bar{\partial}} \lambda=\frac{1}{\lambda}(\bar{\partial}-\mu \partial) \lambda=\frac{1}{\lambda}(\partial \mu) \lambda=\partial \mu$, the 
analogue of $-\frac{1}{2} \int_{\mathbb{C}} d^{2} z \mu \partial^{2} \ln \lambda=\frac{1}{2} \int_{\mathbb{C}} d^{2} z(\partial \mu)(\partial \ln \lambda)=$ $\frac{1}{2} \int_{\mathbb{C}} d^{2} \tilde{z}(\partial \mu)(\tilde{\partial} \ln \lambda)=\frac{1}{2} \int_{\mathbb{C}} d^{2} z(\overline{\tilde{\partial}} \ln \lambda)(\tilde{\partial} \ln \lambda)$ would be

$\Gamma_{W Z P}\left[H_{\bar{\theta}}^{z}, H_{\theta}^{z}\right]=\frac{1}{2} \int_{S \mathbb{C}} d \tilde{z} d \tilde{\bar{z}} d \tilde{\theta} d \tilde{\bar{\theta}}(\tilde{D} \ln \Lambda)(\tilde{\bar{D}} \ln \Lambda)$.

Since the superJacobian of the transformation of the coordinates is $\operatorname{sdet} \frac{\partial(\tilde{z}, \tilde{z}, \tilde{\theta}, \tilde{\tilde{\theta}}}{\partial(z, \bar{z}, \theta, \theta)}=\frac{\sqrt{\Lambda}}{H_{\theta}^{\theta}}$ and $\tilde{D}=\frac{1}{\sqrt{\Lambda} H_{\theta}^{\theta}}\left(D-H_{\theta}^{z} \partial\right)$,

$$
\begin{array}{r}
\Gamma_{W Z P}\left[H_{\bar{\theta}}^{z}, H_{\theta}^{z}\right]=\frac{1}{2} \int_{S \mathbb{C}} d z d \bar{z} d \theta d \bar{\theta} \frac{\sqrt{\Lambda}}{H_{\theta}^{\theta}}\left[\partial H_{\bar{\theta}}^{z}-\frac{H_{\bar{\theta}}^{\theta}}{H_{\theta}^{\theta}} \partial H_{\theta}^{z}\right] \\
\frac{1}{\sqrt{\Lambda} H_{\theta}^{\theta}}\left(D-H_{\theta}^{z} \partial\right) \ln \Lambda \\
=\frac{1}{2} \int_{S \mathbb{C}} d z d \bar{z} d \theta d \bar{\theta} \frac{1}{\left(H_{\theta}^{\theta}\right)^{2}}\left[\partial H_{\bar{\theta}}^{z}-\frac{H_{\bar{\theta}}^{\theta}}{H_{\theta}^{\theta}} \partial H_{\theta}^{z}\right]\left(D-H_{\theta}^{z} \partial\right) \ln \Lambda
\end{array}
$$

The gauge $H_{\theta}^{z}=0,\left(H_{\theta}^{\theta}\right)^{2}=1-\left(D_{\theta}-H_{\theta}^{z} \partial\right) H_{\theta}^{z}=1$ and this integral reduces to

$$
\begin{aligned}
\Gamma_{W Z P}\left[H_{\bar{\theta}}^{z}, H_{\theta}^{z}=0\right] & =\frac{1}{2} \int_{S \mathbb{C}} d z d \bar{z} d \theta d \bar{\theta} \partial H_{\bar{\theta}}^{z} D \ln \Lambda \\
& =-\frac{1}{2} \int_{S \mathbb{C}} d z d \bar{z} d \theta d \bar{\theta} H_{\bar{\theta}}^{z} \partial D \ln \Lambda .
\end{aligned}
$$

The BRST variation of the effective action in this gauge has been computed [6]. With the BRST transformation rules

$$
\begin{aligned}
s H_{\bar{\theta}}^{z} & =\left[D-H_{\bar{\theta}}^{z} \partial+\frac{1}{2}\left(D H_{\bar{\theta}}^{z}\right) D\right] C^{z}+\left(\partial H_{\bar{\theta}}^{z}\right) C^{z} \\
s \Lambda & =C^{z} \partial \Lambda+\frac{1}{2}\left(D C^{z}\right) D \Lambda+\Lambda \partial C^{z}
\end{aligned}
$$

$s \Gamma_{W Z P}\left[H_{\bar{\theta}}^{z}, H_{\theta}^{z}=0\right]=-\frac{1}{2} \int_{S \mathbb{C}} d z d \bar{z} d \theta d \bar{\theta} H_{\bar{\theta}}^{z} \partial D \ln \Lambda$

$-\frac{1}{2} \int_{S \mathbb{C}} d z d \bar{z} d \theta d \bar{\theta} H_{\theta}^{z}(-\partial D) \operatorname{s} \ln \Lambda=$

$=-\frac{1}{2} \int_{S \mathbb{C}} d z d \bar{z} d \theta d \bar{\theta}\left\{\left[-\bar{D}-H_{\bar{\theta}}^{z} \partial-\frac{1}{2}\left(D H_{\bar{\theta}}^{z}\right) D\right] C^{z}\right.$

$\left.+\left(\partial H_{\bar{\theta}}^{z}\right) C^{z}\right\} \partial D \ln \Lambda=$

$+\frac{1}{2} \int_{S \mathbb{C}} d z d \bar{z} d \theta d \bar{\theta} H_{\bar{\theta}}^{z} \partial D \frac{1}{\Lambda}$

$\left(C^{z} \partial \Lambda+\frac{1}{2}\left(D C^{z}\right) D \Lambda+\Lambda \partial C^{z}\right)$

$=\frac{1}{2} \int_{S \mathbb{C}} d z d \bar{z} d \theta d \bar{\theta} C^{z}\left[-\bar{D}-H_{\bar{\theta}}^{z} \partial-\frac{1}{2}\left(D H_{\bar{\theta}}^{z}\right) D\right]$

$\partial D \ln \Lambda$

$-\frac{1}{2} \int_{S \mathbb{C}} d z d \bar{z} d \theta d \bar{\theta}\left(\partial H_{\bar{\theta}}^{z}\right) C^{z} \partial D \ln \Lambda$

$\left.+\frac{1}{2} \int_{S \mathbb{C}} d z d \bar{z} d \theta d \bar{\theta} H_{\bar{\theta}}^{z}\right] \partial D$

$\frac{1}{\Lambda}\left(C^{z} \partial \Lambda+\frac{1}{2}\left(D C^{z}\right) D \Lambda+\Lambda \partial C^{z}\right)$,

because integration by parts in the Grassmann variable does not require a change in the sign, which can be seen, for example, in $\int d \theta\left(\frac{\partial}{\partial \theta}\left(a_{1}+b_{1} \theta\right)\right)\left(a_{2}+b_{2} \theta\right)=$ $b_{1} b_{2}=\int d \theta\left(a_{1}+b_{1} \theta\right) \frac{\partial}{\partial \theta}\left(a_{2}+b_{2} \theta\right)$. Commuting $\bar{D}$ through $\partial D$ introduces a new negative sign since $D \bar{D} y(\theta, \bar{\theta})=$ $-\bar{D} D y(\theta, \bar{\theta})$. Commuting $\left(D H_{\bar{\theta}}\right) D$ through $\partial D$ preserves the sign. However, this term vanishes since $D^{2} y(\theta, \bar{\theta})=0$. Consequently, the relation $\left[\bar{D}-H_{\bar{\theta}}^{z} \partial+\frac{1}{2}\left(D H_{\bar{\theta}}^{z}\right) D\right] \Lambda=$ $\left(\partial H_{\bar{\theta}}^{z}\right) \Lambda[1]$ yields

$$
\begin{aligned}
s \Gamma_{W Z P}= & \frac{1}{2} \int d z d \bar{z} d \theta d \bar{\theta} C^{z} \partial D\left(\frac{1}{\Lambda}\left(\partial H_{\bar{\theta}}^{z}\right) \Lambda\right) \\
+ & \frac{1}{2} \int d z d \bar{z} d \theta d \bar{\theta} H_{\overline{\bar{\theta}}} \partial D \partial C^{z} \\
& + \text { extra terms }
\end{aligned}
$$

Again, integration by parts of the operator $D$ will not affect the sign,

$$
s \Gamma_{W Z P}=\int d z d \bar{z} d \theta d \bar{\theta} C^{z} \partial^{2} D H_{\bar{\theta}}^{z}+\text { extra terms }
$$

The first integral represents the superdiffeomorphism anomaly.

The Wess-Zumino-Polyakov action may be derived for closed Riemann surfaces of arbi-

trary genus [?][3]. Since a Weyl anomaly occurs in the BRST variation of the diffeomorphism invariant effective action $\Gamma_{D}$, counterterms may be added remove this anomaly and replace it with a diffeomorphism anomaly. With the conformal factor written as $\lambda=e^{\varphi} \rho_{0}$,

$$
\begin{aligned}
\Gamma_{W}(\mu, \bar{\mu}, \lambda) & =\Gamma_{D}(\mu, \bar{\mu}, \lambda)+\Delta \Gamma(\mu, \bar{\mu}, \lambda) \\
\Delta_{1}(\mu, \bar{\mu}, \lambda) & =-\frac{1}{2} \int_{\Sigma} d^{2} z\left[\frac{1}{2(1-\mu \bar{\mu})}(\partial-\mu \bar{\partial})\right. \\
\phi & (\bar{\partial}-\mu \partial) \phi-\phi\left[\partial \bar{\partial} l n \rho_{0}-(\partial-\mu \bar{\partial}-(\bar{\partial} \bar{\mu}))\right. \\
& \left.\left.\frac{\nabla_{0} \mu}{1-\mu \bar{\mu}}-(\bar{\partial}-\mu \partial-(\partial \mu)) \frac{\bar{\nabla}_{0} \bar{\mu}}{1-\mu \bar{\mu}}\right]\right\} \\
\Delta_{2} \Gamma(\mu, \bar{\mu}, \lambda) & =\frac{1}{2} \int_{\Sigma} d^{2} z\left\{\mu\left(\mathcal{R}_{0}-r_{0}\right)+\bar{\mu}\left(\bar{R}_{0}-\bar{r}_{0}\right)\right. \\
& -\frac{1}{(1-\mu \bar{\mu})}\left[\nabla_{0} \mu \overline{\nabla_{0}} \bar{\mu}-\frac{1}{2} \bar{\mu}\left(\nabla_{0} \mu\right)^{2}\right. \\
& \left.\left.-\frac{1}{2} \mu\left(\bar{\nabla}_{0} \bar{\mu}\right)^{2}\right]\right\}
\end{aligned}
$$

where $r_{0}=\partial^{2} \ln \rho_{0}-\frac{1}{2}\left(\partial \ln \rho_{0}\right)^{2}$ is a projective connection with the transformation rule $\left(r_{0}\right)_{a}=\left(\partial_{z_{a}} z_{b}\right)^{2}\left[\left(r_{0}\right)_{b}-\right.$ $\left.\left\{z_{a}, z_{b}\right\}\right]$ in the overlap of the coordinate patches $\left.\left\{U_{a}, z_{a}\right)\right\}$ and $\left\{\left(U_{b}, z_{b}\right)\right.$ in $\Sigma$ and $\mathcal{R}_{0}$ is a holomorphic projection satisfying $s \mathcal{R}_{0}=0$ and a coefficient representing the central charge is set equal to 1 [2]. Then

$$
\begin{aligned}
& s \Gamma_{W}\left(\mu, \bar{\mu}, \mathcal{R}_{0}, \overline{\mathcal{R}}_{0}\right)=\int_{\Sigma} d^{2} z c\left(\partial^{3}+2 \mathcal{R}_{0} \partial+\left(\partial \mathcal{R}_{0}\right)\right) \mu \\
& +\int_{\Sigma} d^{2} z \bar{c}\left(\bar{\partial}^{3}+2 \overline{\mathcal{R}}_{0} \bar{\partial}+\left(\bar{\partial} \overline{\mathcal{R}}_{0}\right)\right) \bar{\mu}
\end{aligned}
$$


is the sum of a holomorphic and anholomorphic component. The holomorphic term is the variation of the WessZumino-Polyakov action $\Gamma_{W Z P}$ on the surface $\Sigma$.

Another formula for this action includes

$$
\begin{aligned}
& \frac{1}{2} \int_{\Sigma} d^{2} z\left[2 \mathcal{R}_{0} \mu+2 \partial^{2} \mu+\partial \ln \Omega_{0 D} \partial \mu\right. \\
& \left.\quad+\partial \ln \Omega(\mu)_{D} \partial \mu+\partial \ln \Omega_{0 D} \partial \ln \Omega(\mu)_{D \mu}\right] \\
& \quad+\frac{1}{4 i} \oint_{\partial D\left(\omega_{0}\right)} \ln \left(\frac{\Omega(\mu)_{D}}{\omega_{0}}\right) d \ln \left(\frac{\omega(\mu)}{\Omega_{0 D}}\right) \\
& \quad+\frac{1}{2} \sum_{k} \nu_{0 k} \ln \left(\frac{\omega(\mu)}{\Omega_{0 D}}\right)\left(\hat{P}_{0 k}\right)
\end{aligned}
$$

with $\Omega$ being a polydromic differential that has no zeros on $\Sigma$ replacing the Dif $f_{0}(\Sigma)$ invariant one-forms $\omega$ with $2 g-2$ zeros at $P_{k}(\mu)$ with multiplicity $\nu_{k}(\mu), \hat{P}_{k}$ is the lift of $P_{k}$ to the covering surface and $\omega_{0}$ and $\Omega_{0}$ are reference one-forms. The third integral is necessary as a result of the contribution of these zeros.

The differentials have been constructed to satisfy gluing conditions amongst the coordi-

nate charts with transition functions that are $P S L(2 ; \mathbb{C})$ elements which would be necessary in a planar covering of the Riemann surface. The domain of integration then would be $\mathbb{C} \backslash\left\{\cup_{\ell=1}^{g} D_{T_{\ell}} \cup D_{T_{\ell}^{-1}}, \quad \ell=1, \ldots, g\right\}$. The integral of a differential on this region would equal an integral over the boundary consisting of the isometric circles $\left.I_{T_{\ell}}, I_{T_{\ell}^{-1}}, \ell=1, \ldots, g\right\}$. Given a $P S L(2 ; \mathbb{C})$ invariance, the contour integrals on $I_{T_{\ell}}$ and $I_{T_{\ell}^{-1}}$ would cancel because the integration paths have the opposite orientation. It remains to be established if this cancellation is preserved under BRST transformations.

The generalization of the Polyakov action to superRiemann surfaces of higher genus begins with a direct generalization of the integral for the super-torus that satisfies a superconformal Ward identity for the effective action $\Gamma_{1}\left[R_{z \theta}, H_{\bar{\theta}}^{z}\right]=\frac{1}{4 \pi} \int d^{2} \lambda \hat{A}_{1}$, where $\hat{A}_{1}=4\left(R_{z \theta}-R_{\chi_{0}}\right) H_{\bar{\theta}}^{z}+$ $D_{\theta}\left(\Delta_{\chi}+\Delta_{\chi_{0}}\right) H_{\bar{\theta}}^{z}, \chi=-D_{\theta} \ln \Psi$ and $\chi_{0}=-D_{\theta} \ln \Psi_{0}$ are the superaffine connections derived from the superhalf-differentials $\Psi$ and $\Psi_{0}, R_{z \theta}=-\partial_{z} \zeta_{\theta}-\zeta_{\theta} D_{\theta} \zeta_{\theta}$ is the superprojective connection given in terms of the coefficient of the connection $\zeta_{\theta}=-D_{\theta} \ln D_{\theta} \hat{\Theta}$ constructed from the solution to the super-Beltrami equation for the superprojective structure consisting of charts from the superRiemann surface to $\mathbb{C}^{1 \mid 1}$ with coordinates $\left(\hat{Z}_{\alpha}, \hat{\Theta}_{\alpha}\right)$ and $\left\{H_{\bar{\theta}}^{z}\right\}$ is the set of super-Beltrami coefficients, and several new terms including the integral $\Gamma_{2}\left[H_{\bar{\theta}}^{z}\right]=\frac{1}{2 \pi i} \oint \hat{A}_{2}$ with

$$
\hat{A}_{2}=\ln \left(\frac{\Psi}{\eta_{0}}\right) \hat{d} \ln \left(\frac{\eta}{\Psi_{0}}\right)
$$

must introduced for cancellation of the BRST variation of the integrand and the action of the BRST operator [4] is given by

$s \ln \Psi=\frac{1}{2} \Delta_{\chi} C^{z} \quad s \Psi_{0}=0 \quad s \ln \eta=\frac{1}{2} \Delta_{\xi} C^{z} \quad s \eta_{0}=0$. with $C^{z}$ being the superdiffeomorphism ghost field. The BRST transformation of this term in the effective action therefore yields a contour integral on the super-Riemann surface that must be evaluated through a supersymmetric generalization of Stokes' theorem.

To develop the theory of higher-genus contributions to the supergravity action, it may be recalled that Stokes' theorem has a superspace generalization [4][7]. Let

$$
\begin{aligned}
\hat{d} \Phi & =\left(d \lambda D_{\theta}+(-1)^{p+q} d \hat{\lambda} D_{\bar{\theta}}\right) \Phi \\
\Phi & =\Phi_{\theta} d \lambda \\
\Phi_{\theta}(z, \theta, \bar{z}, \bar{\theta}) & =\phi_{0}+\left(\theta-\theta_{0}\right) \phi_{1}+\left(\bar{\theta}-\bar{\theta}_{0}\right) \phi_{2} \\
& +\left(\theta-\theta_{0}\right)\left(\bar{\theta}-\bar{\theta}_{0}\right) \phi_{3}
\end{aligned}
$$

Then

$D_{\bar{\theta}} \Phi_{\theta}=\phi_{2}-\left(\theta-\theta_{0}\right) \phi_{3}+\left(\tilde{\theta}-\tilde{\theta}_{0}\right) \tilde{\partial} \phi_{0}-\left(\theta-\theta_{0}\right)\left(\tilde{\theta}-\tilde{\theta}_{0}\right) \tilde{\partial} \phi_{0}$

$$
\begin{aligned}
\int_{\hat{D}} \hat{D} \Phi & =-\int_{\hat{D}} d \bar{\lambda} \wedge d \lambda\left(\theta-\theta_{0}\right)\left(\bar{\theta}-\bar{\theta}_{0}\right) \bar{\partial} \phi_{1} \\
& =-\int_{D} d \bar{z} \wedge d z \bar{\partial} \phi_{1}=\oint_{\partial D} \phi_{1} d z
\end{aligned}
$$

and

$$
\begin{aligned}
\oint_{\partial \hat{D}} \Phi & =\oint_{\partial \hat{D}} \Phi_{\theta} d \lambda=\oint_{\partial \hat{D}}\left(\theta-\theta_{0}\right) \phi_{1} d z d \theta \\
& =\oint_{\partial D} \phi_{1} d z .
\end{aligned}
$$

Therefore,

$$
\int_{\hat{D}} \hat{D} \Phi=\oint_{\partial \hat{D}} \Phi
$$

and the super-Stokes theorem is verified.

Theorem 1. The BRST variation of $\hat{A}_{2}$ is given by the sum of $\hat{d}\left[s \ln \eta \ln \left(\frac{\Psi}{\eta_{0}}\right)\right]+d \lambda T_{\lambda}+d \bar{\lambda} \bar{T}_{\bar{\lambda}}$, where $T_{\lambda}=$ $\frac{1}{2} D_{z} \phi+\left(R_{z \theta}-R_{\zeta_{0}}\right) C^{z}-\left(R_{z \theta}-R_{\chi_{0}}\right) C^{z}, \bar{T}_{\bar{\lambda}}=-s \ln \Psi$ $D_{\bar{\theta}} \ln \left(\frac{\eta}{\Psi_{0}}\right)-s \ln \eta D_{\bar{\theta}} \ln \left(\frac{\Psi}{\eta_{0}}\right)$ with $\zeta_{0}=-D_{\theta} \ln \eta_{0}$, $\zeta=-D_{\theta} \ln \eta$ and

$\phi=D_{\theta}\left[C^{z}\left(\chi_{0}+\chi-\zeta_{0}-\zeta\right)\right]+\left(\zeta \zeta_{0}-\chi \chi_{0}-2 \zeta \chi\right) C^{z}$, and three extra terms. Two terms are total derivatives which vanish upon integration over a closed Riemann surface of finite genus. The presence of the third term requires a cancellation of the commutator of the superspace derivative and the BRST transformation.

Proof.

The BRST transformation of the term $\hat{A}_{2}$ equals

$s \hat{A}_{2}=s\left\{\hat{d}\left[\ln \left(\frac{\Psi}{\eta_{0}}\right) \ln \left(\frac{\eta_{0}}{\Psi_{0}}\right)\right]-\left[\hat{d} \ln \left(\frac{\Psi}{\eta_{0}}\right) \ln \left(\frac{\eta}{\Psi_{0}}\right)\right]\right\}$ 
Since the BRST transformation and superspace derivative anticommute, $s \hat{d}+\hat{d} s=0$, then

$$
\left.s \hat{d}\left[\ln \left(\frac{\Psi}{\eta_{0}}\right) \ln \left(\frac{\eta}{\Psi_{0}}\right)\right]-\hat{d} s\left[\ln \left(\frac{\Psi}{\eta_{0}}\right) \ln \left(\frac{\eta}{\Psi_{0}}\right)\right]\right\} \text {. }
$$

and

$$
\begin{aligned}
\hat{d} s\left[\ln \left(\frac{\Psi}{\eta_{0}}\right) \ln \left(\frac{\eta}{\Psi_{0}}\right)\right] & =\hat{d}\left[s \ln \left(\frac{\Psi}{\eta_{0}}\right) \ln \left(\frac{\eta}{\Psi_{0}}\right)\right] \\
& +\hat{d}\left[\ln \left(\frac{\Psi}{\eta_{0}}\right) \ln \left(\frac{\eta}{\Psi_{0}}\right)\right](30) \\
\hat{d}\left[\operatorname{sln}\left(\frac{\eta}{\Psi_{0}}\right) s \ln \Psi\right] & =\operatorname{sln}\left(\frac{\Psi}{\eta_{0}}\right) \\
& \left(D_{\theta}\left(\frac{\eta}{\Psi_{0}}\right) d \lambda-D_{\bar{\theta}} \ln \left(\frac{\eta}{\Psi_{0}}\right) d \bar{\lambda}\right) \\
& +\hat{d} s\left(\frac{\Psi}{\eta_{0}}\right) \ln \left(\frac{\eta}{\Psi_{0}}\right) \\
& -s\left[\hat{d}\left(\frac{\Psi}{\eta_{0}}\right) \ln \left(\frac{\eta}{\Psi_{0}}\right)\right] \\
& =\sin \left(\frac{\eta}{\Psi_{0}}\right)\left[D_{\theta} \ln \left(\frac{\Psi}{\eta_{0}}\right) d \lambda\right. \\
& \left.-D_{\bar{\theta}} \ln \left(\frac{\Psi}{\eta_{0}}\right) d \bar{\lambda}\right] \\
& -s \hat{d} \ln \left(\frac{\Psi}{\eta_{0}}\right) \ln \left(\frac{\eta}{\Psi_{0}}\right) .
\end{aligned}
$$

Amongst the terms in the expression is

$$
\begin{aligned}
-s \ln \left(\frac{\Psi}{\eta_{0}}\right) & D_{\theta} \ln \left(\frac{\eta}{\Psi_{0}}\right) d \lambda-\operatorname{sln}\left(\frac{\Psi}{\eta_{0}}\right) D_{\bar{\theta}} \ln \left(\frac{\eta}{\Psi_{0}}\right) d \bar{\lambda} \\
& -(\hat{d} s+s \hat{d}) \ln \left(\frac{\Psi}{\eta_{0}}\right) \ln \left(\frac{\eta}{\Psi_{0}}\right) \\
& +\operatorname{sln}\left(\frac{\eta}{\Psi_{0}}\right) D_{\theta} \ln \left(\frac{\Psi}{\eta_{0}}\right) d \lambda \\
& +\ln \left(\frac{\eta}{\Psi_{0}}\right) D_{\bar{\theta}} \ln \left(\frac{\Psi}{\eta_{0}}\right) d \bar{\lambda}
\end{aligned}
$$

The remaining terms are

$$
\begin{aligned}
& -s \ln \Psi D_{\theta} \ln \left(\frac{\eta}{\Psi_{0}}\right) d \lambda+\ln \left(\frac{\eta}{\Psi_{0}}\right)(\hat{d} s-s \hat{d} \lambda) \\
& \ln \left(\frac{\Psi}{\eta_{0}}\right)+s \ln \eta D_{\theta} \ln \left(\frac{\Psi}{\eta_{0}}\right) d \lambda
\end{aligned}
$$

Consider

$$
\begin{aligned}
T_{\lambda} & =\frac{1}{2} D_{\theta} \phi+\left(R_{z \theta}-R_{\zeta_{0}}\right) C^{z}-\left(R_{z \theta}-R_{\chi_{0}}\right) C^{z} \\
\phi & =D_{\theta}\left[C^{z}\left(\chi_{0}+\chi-\zeta_{0}-\zeta\right)+\left(\zeta \zeta_{0}-\chi \chi_{0}-2 \zeta \chi\right) C^{z} .\right.
\end{aligned}
$$

Then

$D_{\theta} \phi=D_{\theta}^{2}\left[C^{z}\left(\chi_{0}+\chi-\zeta_{0}-\zeta\right)\right]+D_{\theta}\left[\left(\zeta \zeta_{0}-\chi \chi_{0}-2 \zeta \chi\right) C^{z}\right]$

and, since

$$
\begin{aligned}
\Delta_{\xi}(-\chi & \left.+\zeta_{0}\right)=\left(-\Delta_{\chi_{0}}+\Delta_{\xi}\right)(-\chi)-\Delta_{\chi_{0}} \chi+\left(\Delta_{\xi}-\Delta_{\zeta}\right) \zeta_{0} \\
+\Delta_{\zeta} \zeta_{0} & \\
\Delta_{\chi}\left(\zeta-\chi_{0}\right) & =\left(\Delta_{\chi}-\Delta_{\zeta_{0}}\right) \zeta+\Delta_{\zeta_{0}} \zeta-\Delta_{\chi} \chi_{0}
\end{aligned}
$$

and

$$
\begin{aligned}
\frac{1}{2} \Delta_{\xi}\left(C^{z}\left(-\chi+\zeta_{0}\right)\right) d \lambda & =\left\{\frac{1}{2} D_{\theta}^{2}\left[C^{z}\left(-\chi+\zeta_{0}\right)\right]\right. \\
& \left.-\frac{1}{2} C^{z} \Delta_{\xi}\left(-\chi+\zeta_{0}\right)\right\} d \lambda
\end{aligned}
$$

$-\frac{1}{2} \Delta_{\chi}\left(C^{z}\left(\zeta-\chi_{0}\right)\right) d \lambda=\left\{-\frac{1}{2} D_{\theta}^{2}\left[C^{z}\left(\zeta-\chi_{0}\right)\right]+\frac{1}{2} C^{z} \Delta_{\chi}\left(\zeta-\chi_{0}\right)\right\} d \lambda$

$s \ln \eta D_{\theta} \ln \left(\frac{\Psi}{\eta_{0}}\right) d \lambda-s \ln \Psi D_{\theta} \ln \left(\frac{\eta}{\Psi_{0}}\right) d \lambda$

$$
=\left\{\frac{1}{2} D_{\theta}^{2}\left[C^{z}\left(-\chi+\zeta_{0}+\zeta-\chi_{0}\right)\right]+\left(\Delta_{\chi_{0}}-\Delta_{\xi}\right) \chi\right.
$$$$
+\left(\Delta_{\xi}-\Delta_{\zeta}\right) \zeta_{0}
$$$$
\left.+\left(\Delta_{\xi}-\Delta_{\zeta}\right) \zeta_{0}+\left(\Delta_{\chi}-\Delta_{\zeta_{0}}\right) \zeta-R_{\chi_{0}} C^{z}+R_{\zeta_{0}} C^{z}\right\} d \lambda
$$$$
=\left(-\frac{1}{2} D_{\theta} \phi+R_{\zeta_{0}} C^{z}-R_{\chi_{0}} C^{z}\right) d \lambda
$$$$
+\frac{1}{2} D_{\theta}\left[\left(\zeta \zeta_{0}-\chi \chi_{0}-2 \zeta \chi\right) C^{z}\right] d \lambda
$$$$
+\frac{1}{2}\left[\left(\Delta_{\chi_{0}}-\Delta_{\xi}\right) \chi+\left(\Delta_{\xi}-\Delta_{\zeta}\right) \zeta_{0}+\left(\Delta_{\chi}-\Delta_{\zeta_{0}}\right) \zeta\right] d \lambda
$$$$
=d \lambda\left(\frac{1}{2} D_{\theta} \phi+\left(R_{z \theta}-R_{\zeta_{0}}\right) C^{z}-\left(R_{z \theta}-R_{\chi_{0}}\right) C^{z}\right)
$$$$
+\frac{1}{2} D_{\theta}\left[\left(\zeta \zeta_{0}-\chi \chi_{0}-2 \zeta \chi\right) C^{z}\right] d \lambda
$$$$
+\frac{1}{2}\left[\left(\Delta_{\chi_{0}}-\Delta_{\xi}\right) \chi+\left(\Delta_{\xi}-\Delta_{+} \zeta\right) \zeta_{0}+\left(\Delta_{\chi}-\Delta_{\zeta_{0}}\right) \zeta\right] d \lambda .
$$

It follows that

$T_{\lambda} d \lambda=\sin \eta D_{\theta} \ln \left(\frac{\Psi}{\eta_{0}}\right) d \lambda-\sin \Psi D_{\theta} \ln \left(\operatorname{frac} \eta \Psi_{0}\right) d \lambda$

$T_{\bar{\lambda}} d \bar{\lambda}=\ln \eta D_{\bar{\theta}} \ln \left(\frac{\Psi}{\eta_{0}}\right) d \bar{\lambda}-s \ln \quad D_{\bar{\theta}} \ln \left(\frac{\eta}{\Psi_{0}}\right) d \bar{\lambda}$

Substitution into Eqs.(2.27) and (2.28) gives

$$
\begin{aligned}
s \hat{A}_{2}= & \hat{d}\left[s \ln \eta \ln \left(\frac{\Psi}{\eta_{0}}\right)\right]+d \lambda T_{\lambda}+d \bar{\lambda} \bar{T}_{\bar{\lambda}} \\
& +\frac{1}{2} D_{\theta}\left[\left(\zeta \zeta_{0}-\chi \chi_{0}-2 \zeta \chi\right) C^{z}\right] d \lambda \\
& +\frac{1}{2}\left[\left(\Delta_{\chi_{0}}-\Delta_{\xi}\right) \chi+\left(\Delta_{\xi}-\Delta_{\zeta}\right) \zeta_{0}+\left(\Delta_{\chi}-\Delta_{\zeta_{0}}\right) \zeta\right] d \lambda \\
& +\ln \left(\frac{\eta}{\Psi_{0}}\right)(-\hat{d} s-s \hat{d}) \ln \left(\frac{\Psi}{\eta_{0}}\right) .
\end{aligned}
$$

Three new terms in addition to $\hat{d}\left[s \ln \eta \ln \left(\frac{\Psi}{\eta_{0}}\right)\right]+$ $d \lambda T_{\lambda}+d \bar{\lambda} \bar{T}_{\bar{\lambda}}$ occur in the BRST variation of $\hat{A}_{2}$ [4]. The first term $\frac{1}{2} D_{\theta}\left[\left(\zeta \zeta_{0}-\chi \chi_{0}-2 \zeta \chi\right) C^{z}\right] d \lambda$ is a derivative along the Grassmann variable in superspace, and the integral vanishes on a closed finite-genus super-Riemann surface by the super-Stokes theorem. The second term consists of Laplacians which will integrate to zero on a compact surface. The integral of the third term is

$$
\int_{\hat{D}} \ln \left(\frac{\eta}{\Psi_{0}}\right)(-\hat{d} s-s \hat{d}) \ln \left(\frac{\Psi}{\eta_{0}}\right)
$$


While the superconformal and BRST transformations do not necessarily commute in a supersymmetric gauge theory [5], the vanishing of the anticommutator of $s$ and $\hat{d}$ is sufficient to ensure the absence of a third integral.

The entire set of terms in the effective action has been found to be $\Gamma=\Gamma_{1}+\Gamma_{2}+\Gamma_{3}+\Gamma_{4}$, where $\Gamma_{3}$ is another contour integral $\Gamma_{3}\left[H_{\bar{\theta}}^{z}\right]=\frac{1}{4 \pi i} \oint_{\partial D} d \bar{\lambda}\left[\left(\zeta \zeta_{0}-\right.\right.$ $\left.\left.\chi \chi_{0}-2 \zeta \chi\right) H_{\theta}-\frac{1}{2}\left(\chi+\chi_{0}-\zeta-\zeta_{0}\right) D_{\theta} H_{\bar{\theta}}^{z}\right]$ and $\Gamma_{4}$ is a sum of residues $\Gamma_{4}\left[H_{\bar{\theta}}^{z}\right]=\sum_{k} \frac{\alpha_{0 k}}{4} \triangle_{\zeta} C^{z}\left(P_{0 k}\right)$ with $\eta_{0}\left(P_{k}\right)=\beta\left(P_{k}\right)\left(z_{k}-z_{0 k}-\theta_{k} \theta_{0 k}\right)^{\frac{1}{2} \alpha_{0 k}}$ [4]. The variations of $\Gamma_{2}$ and $\Gamma_{3}$ cancel extra terms in $s \Gamma_{1}\left[R_{z \theta}, H_{\bar{\theta}}^{z}\right]$, leaving only a globally defined anomaly. Therefore, the vanishing of the new terms introduced in Theorem 1 is necessary for a consistent formulation of the effective action. The third contour integral does not vanish immediately by the super-Stokes theorem. Nevertheless, the dissection into a $4 g$-sided polygon which contains no zeros of $\eta$ is sufficient to establish that the integral over contours surrounding the points $P_{0 k}$ will vanish again by the residue theorem $[3]$.

Superconformal classes of metrics on a super-Riemann surface are parameterized by $H^{z}{ }_{\bar{\theta}}, H^{z}$. However, the superconformal transformation mixes both $H_{\bar{\theta}}^{z}$ and $H^{z}{ }_{\theta}$, complicating the description of supermoduli space. This result reflects the non-splitness of supermoduli space. It is known also that, with a super-projective connection $\mathcal{R}(z, \theta)$, the modified anomaly $\tilde{A}\left(C^{z} ; H_{\bar{\theta}}^{z}\right)=C^{z}(\partial D+$ $3 \mathcal{R}_{z \theta} \partial+\left(D \mathcal{R}_{z \theta) D+2\left(\partial \mathcal{R}_{z \theta}\right)} H_{\bar{\theta}}^{z}\right.$ transforms with the superjacobian under superconformal transformations [6].

There does exist a formulation of nonperturbative quantum chromodynamics with phantom symmetries such that a generalized momentum operator does not commute with the BRST charge [8]. Since the effects are nonperturbative, these commutators would not be related to induced supergravity actions at finite genus. At infinite genus, there will be a new contribution to the contour integrals from the ideal boundary. It is necessary to establish that the contour integrals of the BRST variation over the ideal boundary vanish. This result may be achieved either by a restriction to the class of Type I surfaces or a quasiconformally invariant subset or field that have BRST variation that tends to zero at the boundary.

\section{The Induced Supergravity Action at Infinite Genus}

Additional terms in the induced supergravity action occur when the action of the BRST transformation is nonvanishing. Consider a surface with an ideal boundary of zero linear measure. These Type I surfaces have the classes $O_{G}$, characterized by zero harmonic measure and no Green function with a single source, and $O_{A B}$, that have no nonconstant, bounded, analytic functions, as subsets. However, since each end of a Riemann surface is identified with one point of the ideal boundary, it is necessary to consider the contour integral over the actual boundary arc. For example, the integral over a circular boundary at an end of a surface would be non-zero. The commutation of the conformal and BRST transformations therefore will be proven to be required for the class of $O_{G}$ surfaces.

Theorem 2. The BRST contour integrals of fields on an $O_{G}$ surface of infinite genus vanish on the ideal boundaries if the BRST transformation and the conformal transformations commute.

\section{Proof.}

The BRST contour integral over the commutator of the BRST operator with a vertex operator may be defined on the ideal boundary or the boundary of the end of a surface. Since the fields satisfy classical Laplacian equations on the surface, the addition of a harmonic or analytic function represents only another solution. Nevertheless, the absence of nonconstant harmonic functions with finite Dirichlet integrals in $O_{H D}$, nonconstant analytic functions with finite Dirichlet integrals in $O_{A D}$ and bounded nonconstant analytic functions on an $O_{A B}$ surface, and the conformal invariance of the classification of Riemann surfaces of infinite genus, precludes the addition of arbitrary harmonic or analytic functions to the integrands of the BRST integrals. Consequently, given a meromorphic function that tends to zero at the boundary of an end of infinite extent, the BRST contour integral would not change because the Dirichlet integral can be converted into a contour integral by Stokes' theorem. The contour integral also vanishes for the conformally transformed surface with the boundary given by the accumulation point of an infinite sequence of handles. Similarly, for the supersymmetric theory, as a result of the super-Stokes theorem, the integral of the superspace derivative of a function on the super-Riemann surface would be equal to the the integral of the function over the contour on the ideal boundary. However, since the super-differential and the BRST transformation do not commute, the BRST transformation of a superspace derivative of a function cannot be integrated to a boundary term.

It is necessary therefore to establish the equivalence of the evaluation of integrals of fields that tend to zero at boundaries of surfaces of infinite extent with the vanishing of the integral over a discrete set of accumulation points of handles on a sphere with zero linear measure. A field which tends to zero at infinity can have finite, analytic limits at the accumulation points on a sphere of finite extent.

Specifically, the conformal transformation $\phi: z \rightarrow w(z)$, where $z$ is a coordinate tending to $\infty$ on the surface of infinite extent and $w(z)$ has finite values on the surface constructed by attaching handles to the sphere, it follows that any field $\psi$ would be transformed to

$$
\psi^{\prime}(w)=\psi^{\prime}(\phi(z))=\psi(z)
$$


These surfaces can be embedded in $\mathbb{R}^{3}$ through the homeomorphisms $\varphi_{i}$ in an atlas $\left\{\left(U_{i}, \varphi_{i}\right)\right\}$, and, by the extreme value theorem, the set of values of $\left.\psi^{\prime}\right|_{U_{i}}=\eta^{\prime} \circ \varphi_{i}$ would be finite on a compact set in $\mathbb{C}$.

The coordinate $z$ on the surface of infinite extent is not necessarily global. However, the unit disk is a universal cover for both surfaces. Suppose that the coordinate on the unit disk is $t$ such that the boundary is $|t|=1$. Suppose that the projection map from the unit disk to the surface of finite extent is $\pi: D \rightarrow \Sigma_{\text {fin }}$. Then the function $\psi_{D}$ can be defined to be

$$
\psi_{D}(t)=\psi^{\prime}(w)=\psi^{\prime}(\pi(t))
$$

The ideal boundary on the unit disk will be intersection of the fundamental domain with the unit circle consisting of a discrete set of points with zero Lebesgue measure. Then

$$
\int_{\beta} \psi_{D}(t) d t=0
$$

It may be recalled that

$$
\int_{\partial \Sigma_{\text {inf }}} \psi(z) d z=\int_{\beta} \frac{d z}{d w} \frac{d w}{d t} \psi_{D}(t) d t .
$$

The surface $\Sigma_{\text {inf }}$ can be covered by open sets that may be mapped homeomorphically to open sets in the upper half plane by a bounded mapping $\eta$, and the image of the boundary would be located at infinity in $\mathbb{H}$. The conformal mapping $\phi^{-1} \circ \pi$ then may be chosen to be $\phi^{-1} \circ \pi(t)=$ $i \frac{1+t}{1-t}$. Since

$$
\begin{aligned}
\frac{d \phi^{-1} \circ \pi(t)}{d t} & =i \frac{d}{d t}\left(\frac{1+t}{1-t}\right) \\
& =\frac{2 i}{(1-t)^{2}},
\end{aligned}
$$

the integral (3.4) will vanish if $\psi_{D}(t)$ tends to zero as rapidly as $(1-t)^{2}$. It follows that the fields $\psi$ will yield a vanishing integral over the boundary $\partial \Sigma_{\text {inf }}$. when $\psi(z) \rightarrow$ 0 as $\frac{1}{z^{\alpha}}$ with $\alpha \geq 2$.

There is a distinction between the linear measure of the ideal boundary and the border arc of a Riemann surface over which a contour integral may be evaluated. Each end of a surface may be identified with a point on the ideal boundary. A point has no linear measure, and therefore, an integral over this set would equal zero. Yet, the border arc of one end of an infinitely long surface actually has non-zero measure giving rise to a nonvanishing contour integral. Surfaces belong to the class $O_{A B}$ have ideal boundaries of zero linear measure, and yet, this property is not sufficient to ensure a vanishing contour integral. Instead, it is necessary to restrict the category in Theorem 2 to the class of $O_{G}$ surfaces. The rapid decrease of the size of the handles tending towards infinity is sufficient to give a boundary yielding a contour integral.

\section{BRST and Conformal Symmetries in Four Dimensional Gravity}

The relation between between these two symmetries may be considered in four dimensions. The consistency of the projection of the commutation of conformal and BRST transformations reveals the connection between the theories in two and four dimensions. First, it is known that the commutator of BRST transformations in gravitational theories with translation generators on the background vanishes [10]. For conformal transformations, consider the classical gravitational Lagrangian after integration by parts

$$
\begin{aligned}
\mathcal{L}_{c l} & =\frac{2}{\tilde{\kappa}^{2}} \sqrt{-g} g^{\mu \nu}\left(\Gamma_{\mu \nu}^{\rho} \Gamma_{\rho \lambda}^{\lambda}-\Gamma_{\mu \rho}^{\lambda} \Gamma_{\nu \lambda}^{\rho}\right) \\
& =\frac{1}{2 \tilde{\kappa}^{2}}\left[\tilde{g}^{\mu \nu} \tilde{g}_{\lambda \rho} \tilde{g}_{\tau \sigma}-2 \delta_{\tau}{ }^{\nu} \delta_{\lambda}^{\mu} \tilde{g}_{\rho \sigma}-\frac{1}{2} \tilde{g}^{\mu \nu} \tilde{g}_{\rho \tau} \tilde{g}_{\lambda \sigma}\right] \\
& \left(\partial_{\mu} \tilde{g}^{\rho \tau}\right)\left(\partial_{\nu} \tilde{g}^{\rho \sigma}\right) \\
\tilde{g}^{\mu \nu} & =g^{\mu \nu} \sqrt{-g} \\
\tilde{\kappa}^{2} & =32 \pi G,
\end{aligned}
$$

and gauge fixing generates Faddeev-Popov ghosts, which are described by the Lagrangian [10]

$$
\mathcal{L}_{F P}=-\frac{1}{\tilde{\kappa}^{2}}\left[\left(\partial_{\mu} b_{\nu}\right) \tilde{g}^{\mu \nu}+i\left(\partial_{\mu} \bar{c}_{\nu}\right) \delta \tilde{g}^{\mu \nu}-\frac{\alpha}{2} \eta^{\mu \nu} b_{\mu} b_{\nu}\right]
$$

Under a coordinate transformation $x^{\mu}$ to $x^{\prime \mu}$ and $x^{\prime \lambda}=$ $\left(\delta_{\mu}{ }^{\lambda}+\partial_{\mu} f^{\lambda}\right) x^{\lambda}$

$$
\begin{aligned}
\delta_{f}\left(\mathcal{L}_{c l}\right) & =\partial_{\mu} \Lambda_{f, c l}^{\mu} \\
\Lambda_{f, c l}^{\mu} & =-\frac{2}{\tilde{\kappa}^{2}}\left[f^{\mu} \tilde{g}^{\lambda \sigma} R_{\lambda \sigma}+\delta_{f}\left(\tilde{g}^{\mu \lambda} \Gamma_{\lambda \rho}^{\rho}\right)-\delta_{f}\left(\tilde{g}^{\lambda \rho} \Gamma_{\lambda \rho}^{\mu}\right)\right]
\end{aligned}
$$

If $\delta \bar{c}_{\nu}=i b_{\nu}$ and $\delta b_{\nu}=0$

$$
\begin{aligned}
\delta \mathcal{L}_{F P} & =-\frac{1}{\tilde{\kappa}^{2}}\left[\left(\partial_{\mu} \delta b_{\nu}\right) \tilde{g}^{\mu \nu}+\left(\partial_{\mu} b_{\nu}\right) \delta \tilde{g}^{\mu \nu}+i\left(\partial_{\mu} \delta \bar{c}_{\nu}\right) \delta \tilde{g}^{\mu \nu}-\right. \\
& \left.\frac{\alpha}{2} \eta^{\mu \nu} \cdot 2 \delta b_{\mu} b_{\nu}\right] \\
& =-\frac{1}{\tilde{\kappa}^{2}}\left[\left(\partial_{\mu} b_{\nu}\right) \delta \tilde{g}^{\mu \nu}+i\left(i \partial_{\mu} b_{\nu}\right) \delta \tilde{g}^{\mu \nu}\right] \\
& =0
\end{aligned}
$$

The current is given by

$$
\begin{aligned}
\frac{1}{\tilde{\kappa}^{2}} J_{B}^{\mu}= & \frac{\partial \mathcal{L}}{\partial\left(\partial_{\mu} \tilde{g}^{\lambda \sigma}\right)} \delta\left(\tilde{g}^{\lambda \sigma}\right)+\frac{\partial \mathcal{L}}{\partial\left(\partial_{\mu} b_{\lambda}\right)} \delta\left(b_{\lambda}\right) \\
& +\frac{\partial \mathcal{L}}{\partial\left(\partial_{\mu} c^{\lambda}\right)} \delta\left(c^{\lambda}\right)+\frac{\partial \mathcal{L}}{\partial\left(\partial_{\mu} \bar{c}_{\lambda}\right)} \delta\left(\bar{c}_{\lambda}\right)-\Lambda_{f}^{\mu}
\end{aligned}
$$


and

$$
\begin{aligned}
\frac{1}{\tilde{\kappa}^{2}} \partial_{\mu} J_{B}^{\mu} & =\partial_{\mu}\left(\frac{\partial \mathcal{L}}{\partial\left(\partial_{\mu} \tilde{g}^{\lambda \sigma}\right.} \delta\left(\tilde{g}^{\lambda \sigma}\right)\right)+\partial_{\mu}\left(\frac{\partial \mathcal{L}_{F P}}{\partial\left(\partial_{\mu} b_{\lambda}\right)} \delta b^{\lambda}\right) \\
& +\partial_{\mu}\left(\frac{\partial \mathcal{L}_{F P}}{\partial\left(\partial_{\mu} c^{\lambda}\right)} \delta c^{\lambda}\right) \\
& +\partial_{\mu}\left(\frac{\partial \mathcal{L}_{F P}}{\partial\left(\partial_{\mu} \bar{c}_{\lambda}\right)} \delta \bar{c}_{\lambda}\right)-\partial_{\mu} \Lambda_{f}^{\mu} \\
& =\frac{\partial \mathcal{L}}{\partial\left(\tilde{g}^{\lambda \sigma}\right)} \delta \tilde{g}^{\lambda \sigma}+\frac{\partial \mathcal{L}}{\partial\left(\partial_{\mu} g^{\lambda \sigma}\right)} \delta\left(\partial_{\mu} \tilde{g}^{\lambda \sigma}\right. \\
& +\frac{\partial \mathcal{L}}{\partial b_{\lambda}} \delta b_{\lambda}+\frac{\partial \mathcal{L}}{\partial\left(\partial_{\mu} b_{\lambda}\right)} \delta\left(\partial_{\mu} b_{\lambda}\right) \\
& +\frac{\partial \mathcal{L}}{\partial c^{\lambda}} \delta c^{\lambda}+\frac{\partial \mathcal{L}}{\partial\left(\partial_{\mu} c^{\lambda}\right)} \delta\left(\partial_{\mu} c^{\lambda}\right)+\frac{\partial \mathcal{L}}{\partial \bar{c}_{\lambda}} \delta \bar{c}_{\lambda} \\
& +\frac{\partial \mathcal{L}}{\partial\left(\partial_{\mu} \bar{c}_{\lambda}\right)} \delta\left(\partial_{\mu} \bar{c}_{\lambda}\right)-\partial_{\mu} \Lambda_{f}^{\mu}
\end{aligned}
$$

by the Euler-Lagrange equation. Given the formal identity $\delta \mathcal{L}=\partial_{\mu} \Lambda_{f}^{\mu}$, the divergence of the current would vanish.

Given that $\delta_{f}(\sqrt{-g})=-\partial_{\lambda}\left(f^{\lambda} \sqrt{-g}\right)$,

$$
\begin{aligned}
\delta_{f}\left(\tilde{g}^{\mu \nu}\right) & =\delta_{f}\left(g^{\mu \nu} \sqrt{-g}\right)=\left(\delta_{f} g^{\mu \nu}\right) \sqrt{-g}+g^{\mu \nu} \delta_{f}(\sqrt{-g}) \\
& =\left[\left(\partial_{\lambda} f^{\mu}\right) g^{\lambda \nu}+\left(\partial_{\lambda} f^{\nu}\right) g^{\mu \lambda}\right. \\
& \left.-f^{\lambda} \partial_{\lambda} g^{\mu \nu}\right] \sqrt{-g}-g^{\mu \nu} \partial_{\lambda}\left(f^{\lambda} \sqrt{-g}\right) \\
& =\left(\partial_{\lambda} f^{\mu}\right) \tilde{g}^{\lambda \nu}+\left(\partial_{\lambda} f^{\nu}\right) \tilde{g}^{\mu \lambda}-f^{\lambda} \partial_{\lambda} \tilde{g}^{\mu \nu}
\end{aligned}
$$

Let $f^{\mu}=c^{\mu}$ since $c$ can be regarded as a vector field and a generator of a coordinate transformation in a superspace consisting of space-time and spinor coordinates. Then,

$$
\begin{aligned}
& \mathcal{L}_{F P}=-\frac{1}{\tilde{\kappa}^{2}}\left[\left(\partial_{\mu} b_{\nu}\right) \tilde{g}^{\mu \nu}+i\left(\partial_{\mu} \bar{c}_{\nu}\right)\left(\left(\partial_{\lambda} c^{\mu}\right) \tilde{g}^{\lambda \nu}\right.\right. \\
&\left.\left.+\left(\partial_{\lambda} c^{\nu}\right) \tilde{g}^{\mu \lambda}-c^{\lambda} \partial_{\lambda} \tilde{g}^{\mu \nu}\right)-\frac{\alpha}{2} \eta^{\mu \nu} b_{\mu} b_{\nu}\right] \\
& \frac{\partial \mathcal{L}}{\partial \partial_{\mu} b_{\lambda}}=-\frac{1}{\tilde{\kappa}^{2}} \tilde{g}^{\mu \lambda} \\
& \frac{\partial \mathcal{L}}{\partial \partial_{\mu} c^{\lambda}}=\frac{i}{\tilde{\kappa}^{2}}\left[\left(\partial_{\lambda} \bar{c}_{\nu}\right) \tilde{g}^{\mu \nu}+\left(\partial_{\sigma} \bar{c}_{\lambda}\right) \tilde{g}^{\sigma \mu}\right] \\
& \frac{\partial \mathcal{L}}{\partial \partial_{\mu} \bar{c}_{\lambda}}=-\frac{i}{\tilde{\kappa}^{2}} \delta \tilde{g}^{\mu \lambda} .
\end{aligned}
$$

Then

$$
\begin{aligned}
& \frac{\partial \mathcal{L}_{F P}}{\partial \partial_{\mu} b_{\lambda}} \delta b_{\lambda}+\frac{\partial \mathcal{L}_{F P}}{\partial \partial_{\mu} c^{\lambda}} \delta c^{\lambda}+\frac{\partial \mathcal{L}_{F P}}{\partial \partial_{\mu} \bar{c}_{\lambda}} \\
& =\frac{1}{\tilde{\kappa}^{2}} \delta \tilde{g}^{\mu \lambda} b_{\lambda}+\frac{i}{\tilde{\kappa}^{2}}\left[\left(\partial_{\lambda} \bar{c}_{\nu}\right) \tilde{g}^{\mu \nu}+\left(\partial_{\sigma} \bar{c}_{\lambda}\right) \tilde{g}^{\sigma \mu}\right] \delta c^{\lambda} \\
& \frac{1}{\tilde{\kappa}^{2}} \delta_{B R S T}\left(\tilde{g}^{\mu \lambda}\right) b_{\lambda}=\frac{1}{\tilde{\kappa}^{2}}\left[\left(\partial_{\rho} c^{\mu}\right) \tilde{g}^{\lambda \nu} b_{\lambda}\right. \\
& \left.+\left(\partial_{\rho} c^{\lambda}\right) \tilde{g}^{\mu \rho} b_{\lambda}-c^{\rho} \partial_{\rho} \tilde{\partial}^{\mu \lambda} b_{\lambda}\right] .
\end{aligned}
$$

Furthermore,

$$
\begin{aligned}
\frac{\partial \mathcal{L}_{F P}}{\partial \tilde{g}^{\lambda \sigma}} & =-\frac{1}{\tilde{\kappa}^{2}}\left[\partial_{\lambda} b_{\sigma}+i\left(\left(\partial_{\mu} \bar{c}_{\sigma}\right)\left(\partial_{\lambda} c^{\mu}\right)+\left(\partial_{\lambda} \bar{c}_{\nu}\right)\left(\partial_{\sigma} c^{\nu}\right)\right)\right] \\
\frac{\partial \mathcal{L}_{F P}}{\partial \partial_{\mu} \tilde{g}^{\lambda \sigma}} & =\frac{i}{\tilde{\kappa}^{2}}\left(\partial_{\lambda} \bar{c}_{\sigma}\right) c^{\mu}
\end{aligned}
$$

and

$$
\begin{aligned}
& \frac{1}{\tilde{\kappa}^{2}} \delta_{B R S T}\left(\tilde{g}^{\mu \lambda}\right) b_{\lambda}+\frac{1}{\tilde{\kappa}^{2}} \frac{\partial \mathcal{L}_{F P}}{\partial \partial_{\mu} \tilde{g}^{\lambda \sigma}} \delta_{B R S T} \tilde{g}^{\lambda \sigma} \\
& =\frac{1}{\tilde{\kappa}^{2}}\left[\left(\partial_{\rho} c^{\mu}\right) \tilde{g}^{\rho \lambda} b_{\lambda}+\left(\partial_{\rho} c^{\lambda}\right) \tilde{g}^{\mu \rho} b_{\lambda}-c^{\rho} \partial_{\rho} \tilde{\partial}^{\mu \lambda} b_{\lambda}\right] \\
& \quad-\frac{i}{\tilde{\kappa}^{2}}\left[\left(\partial_{\lambda} \bar{c}_{\nu}\right) \tilde{g}^{\mu \nu}+\left(\partial_{\sigma} \bar{c}_{\lambda}\right) \tilde{g}^{\sigma \mu}\right] c^{\rho} \partial_{\rho} c^{\lambda} \\
& \quad+\frac{i}{\tilde{\kappa}^{2}}\left(\partial_{\lambda} \bar{c}_{\sigma}\right) c^{\mu}\left[\left(\partial_{\rho} c^{\lambda}\right) \tilde{g}^{\rho \sigma}+\left(\partial_{\rho} c^{\sigma}\right) \tilde{g}^{\lambda \rho}-c^{\rho} \partial_{\rho} \tilde{g}^{\lambda \sigma}\right]
\end{aligned}
$$

Similarly,

$$
\begin{aligned}
\frac{\partial \mathcal{L}_{c l}}{\partial\left(\partial_{\mu} \tilde{g}^{\gamma \delta}\right)} & =\frac{1}{\tilde{\kappa}^{2}}\left[\tilde{g}^{\mu \nu} \tilde{g}_{\lambda \gamma} \tilde{g}_{\delta \sigma}-2 \delta_{\lambda}^{\mu} \delta_{(} \delta^{\nu} \tilde{g}_{\gamma) \sigma}\right. \\
& \left.-\frac{1}{2} \tilde{g}^{\mu \nu} \tilde{g}_{\gamma \delta} \tilde{g}_{\lambda \sigma}\right] \partial_{\nu} \tilde{g}^{\sigma \lambda} \\
\frac{\partial \mathcal{L}_{c l}}{\partial\left(\partial_{\mu} \tilde{g}^{\lambda \sigma}\right)} \delta\left(\tilde{g}^{\lambda \sigma}\right) & =\frac{1}{\tilde{\kappa}^{2}}\left[\tilde{g}^{\mu \nu} \tilde{g}_{\lambda \gamma} \tilde{g}_{\delta \sigma}-2 \delta_{\lambda}^{\mu} \delta_{(\delta}{ }^{\nu} \tilde{g}_{\gamma) \sigma}-\right. \\
& \left.\frac{1}{2} \tilde{g}^{\mu \nu} \tilde{g}_{\gamma \delta} \tilde{g}_{\lambda \sigma}\right] \partial_{\nu} \tilde{g}^{\sigma \lambda} \\
& {\left[\left(\partial_{\rho} c^{\gamma}\right) \tilde{g}^{\rho \delta}+\left(\partial_{\rho} c^{\delta}\right) \tilde{g}^{\gamma \rho}-c^{\rho} \partial_{\rho} \tilde{g}^{\gamma \delta}\right] }
\end{aligned}
$$

The remaining term is

$$
\Lambda_{c}^{\mu}=-\frac{2}{\tilde{\kappa}^{2}}\left[c^{\mu} \tilde{g}^{\lambda \sigma} R_{\lambda \sigma}+\delta_{c}\left(\tilde{g}^{\mu \lambda} \Gamma_{\lambda \rho}^{\rho}\right)-\delta_{c}\left(\tilde{g}^{\lambda \rho} \Gamma_{\lambda \rho}^{\mu}\right)\right] .
$$

Since $\Gamma_{\lambda \rho}^{\rho}=\frac{1}{\sqrt{-g}} \partial_{\lambda}(\sqrt{-g})$,

$$
\begin{aligned}
& \delta_{B R S T}\left(\tilde{g}^{\mu \lambda} \Gamma_{\lambda \rho}^{\rho}\right)=\delta\left(g^{\mu \lambda} \partial_{\lambda}(\sqrt{-g})\right) \\
& =\left[\left(\partial_{\rho} c^{\mu}\right) g^{\rho \lambda}+\left(\partial_{\rho} c^{\lambda}\right) g^{\mu \rho}-c^{\rho} \partial_{\rho} g^{\mu \lambda}\right] \partial_{\lambda}(\sqrt{-g}) \\
& +g^{\mu \lambda} \partial_{\lambda}\left[-\partial_{\rho}\left(c^{\rho} \sqrt{-g}\right)\right]
\end{aligned}
$$

and

$$
\begin{aligned}
& \delta_{B R S T}\left(\tilde{g}^{\lambda \rho} \Gamma^{\mu}{ }_{\lambda \rho}\right)=\left[\left(\partial_{\sigma} c^{\lambda}\right) \tilde{g}^{\sigma \rho}+\left(\partial_{\sigma} c^{\rho}\right) \tilde{g}^{\lambda \sigma}-c^{\sigma} \partial_{\sigma} \tilde{g}^{\lambda \rho}\right] \Gamma_{\lambda \rho}^{\mu} \\
& +\tilde{g}^{\lambda \rho} \delta_{c}\left[\frac{1}{2} g^{\mu \tau}\left(\partial_{\rho} g_{\tau \lambda}+\partial_{\lambda} g_{\tau \rho}-\partial_{\tau} g_{\lambda \rho}\right)\right] \\
& =\left[\left(\partial_{\sigma} c^{\lambda}\right) \tilde{g}^{\sigma \rho}+\left(\partial_{\sigma} c^{\rho}\right) \tilde{g}^{\lambda \sigma}-c^{\sigma} \partial_{\sigma} \tilde{g}^{\lambda \rho}\right] \Gamma^{\mu}{ }_{\lambda \rho} \\
& +\frac{1}{2} \tilde{g}^{\lambda \rho}\left[\left(\partial_{\sigma} c^{\mu}\right) g^{\sigma \tau}+\left(\partial_{\sigma} c^{\tau}\right) g^{\mu \sigma}-c^{\sigma} \partial_{\sigma} g^{\mu \tau}\right] \\
& \left(\partial_{\lambda} g_{\tau \rho}+\partial_{\tau} g_{\lambda \rho}-\partial_{\rho} g_{\tau \lambda}\right) \\
& -\frac{1}{2} \tilde{g}^{\lambda \rho} g^{\mu \tau} \partial_{\rho}\left(\partial_{\tau} c^{\sigma} g_{\sigma \lambda}+\partial_{\lambda} c^{\sigma} g_{\tau \sigma}+c^{\sigma} \partial_{\sigma} g_{\tau \lambda}\right) \\
& -\frac{1}{2} \tilde{g}^{\lambda \rho} g^{\mu \tau} \partial_{\lambda}\left(\partial_{\tau} c^{\sigma} g_{\sigma \rho}+\partial_{\rho} c^{\sigma} g_{\tau \rho}+c^{\sigma} \partial_{\sigma} g_{\tau \rho}\right) \\
& +\frac{1}{2} g^{\mu \tau} g^{\mu \tau} \partial_{\tau}\left(\partial_{\rho} c^{\sigma} g_{\sigma \lambda}+\partial_{\lambda} c^{\sigma} g_{\rho \sigma}+c^{\sigma} \partial_{\sigma} g_{\lambda \rho}\right) \\
& =\left[\left(\partial_{\sigma} c^{\lambda}\right) \tilde{g}^{\sigma \rho}+\left(\partial_{\sigma} c^{\rho}\right) \tilde{g}^{\lambda \sigma}-c^{\sigma} \partial_{\sigma} \tilde{g}^{\lambda \rho}\right] \Gamma^{\mu}{ }_{\lambda \rho} \\
& -\frac{1}{2} \tilde{g}^{\lambda \rho} g^{\mu \tau}\left(\partial_{\tau} \partial_{\rho} c^{\sigma} g_{\sigma \lambda}+\partial_{\tau} \partial_{\lambda} c^{\sigma} g_{\sigma \rho}+c^{\sigma} \partial_{\tau} \partial_{\sigma} g_{\lambda \rho}\right)
\end{aligned}
$$


The following equations

$$
\begin{aligned}
4 R_{\mu \nu} & =\partial_{\mu} b_{\nu}+\partial_{\nu} b_{\mu}+i\left(\partial_{\mu} \bar{c}_{\lambda}\right)\left(\partial_{\nu} c^{\lambda}\right) \\
& +i\left(\partial_{\nu} \bar{c}_{\lambda}\right)\left(\partial_{\mu} c^{\lambda}\right) \\
& +i \partial_{\nu}\left[\left(\partial_{\lambda} \bar{c}_{\mu}\right) c^{\lambda}\right]+i \partial_{\mu}\left[\left(\partial_{\lambda} \bar{c}_{\nu}\right) c^{\lambda}\right] \\
\partial_{\mu} \tilde{g}^{\mu \nu}+\alpha b^{\nu} & =0 .
\end{aligned}
$$

are derived from the action. Then

$$
\begin{aligned}
\Lambda_{c}^{\mu} & =-\frac{2}{\tilde{\kappa}^{2}}\left[\frac { 1 } { 4 } c ^ { \mu } \tilde { g } ^ { \lambda \sigma } \left\{\partial_{\lambda} b_{\sigma}+\partial_{\lambda} b_{\sigma}+i\left(\partial_{\lambda} \bar{c}_{\rho}\right)\left(\partial_{\sigma} c^{\rho}\right)\right.\right. \\
& \left.+i\left(\partial_{\sigma} \bar{c}_{\rho}\right)\left(\partial_{\lambda} c^{\rho}\right)\right) \\
& \left.+i \partial_{\sigma}\left[\left(\partial_{\rho} \bar{c}_{\lambda}\right) c^{\rho}\right]+i \partial_{\lambda}\left[\left(\partial_{\rho} \bar{c}_{\sigma}\right) c^{\rho}\right]\right\} \\
& +\left[\left(\partial_{\rho} c^{\mu}\right) g^{\rho \lambda}+\left(\partial_{\rho} c^{\lambda}\right) g^{\mu \rho}-c^{\rho} \partial_{\rho} g^{\mu \lambda}\right] \partial_{\lambda}(\sqrt{-g}) \\
& +g^{\mu \lambda} \partial_{\lambda}\left[-\partial_{\rho}\left(c^{\rho} \sqrt{-g}\right)\right] \\
& +\left[\left(\partial_{\sigma} c^{\lambda}\right) \tilde{g}^{\sigma \rho}+\left(\partial_{\sigma} c^{\rho}\right) \tilde{g}^{\lambda \sigma}-c^{\sigma} \partial_{\sigma} \tilde{g}^{\lambda \rho}\right] \Gamma_{\lambda \rho}^{\mu} \\
& -\frac{1}{2} \tilde{g}^{\lambda \rho} g^{\mu \tau}\left(\partial_{\tau} \partial_{\rho} c^{\sigma} g_{\sigma \lambda}+\partial_{\tau} \partial_{\lambda} c^{\sigma} g_{\sigma \rho}+c^{\sigma} \partial_{\tau} \partial_{\sigma} g_{\lambda \rho}\right)(6.7)
\end{aligned}
$$

Combining Eqs.(4.14), (4.15) and (4.20),

$$
\begin{aligned}
J_{B}^{\mu} & =\left[\left(\partial_{\rho} c^{\mu}\right) \tilde{g}^{\rho \lambda} b_{\lambda}+\left(\partial_{\rho} c^{\lambda}\right) \tilde{g}^{\mu \rho} b_{\lambda}-c^{\rho} \partial_{\rho} \tilde{g}^{\mu \lambda} b_{\lambda}\right] \\
& -i\left[\left(\partial_{\lambda} \bar{c}_{\nu}\right) \tilde{g}^{\mu \nu}+\left(\partial_{\sigma} \bar{c}_{\lambda}\right) \tilde{g}^{\sigma \mu}\right] c^{\rho} \partial_{\rho} c^{\lambda} \\
& +i\left(\partial_{\lambda} \bar{c}_{\sigma}\right) c^{\mu}\left[\left(\partial_{\rho} c^{\lambda}\right) \tilde{g}^{\rho \sigma}+\left(\partial_{\rho} c^{\sigma}\right) \tilde{g}^{\lambda \rho}-c^{\rho} \partial_{\rho} \tilde{g}^{\lambda \sigma}\right] \\
& +\left[\tilde{g}^{\mu \nu} \tilde{g}_{\lambda \gamma} \tilde{g}_{\delta \sigma}-2 \delta_{\lambda}^{\mu} \delta\left(\delta^{\nu} \tilde{g}_{\gamma) \sigma}-\frac{1}{2} \tilde{g}^{\mu \nu} \tilde{g}_{\gamma \delta} \tilde{g}_{\lambda \sigma}\right] \partial_{\nu} \tilde{g}^{\sigma \lambda}\right. \\
& {\left[\left(\partial_{\rho} c^{\gamma}\right) \tilde{g}^{\rho \delta}+\left(\partial_{\rho} c^{\delta}\right) \tilde{g}^{\gamma \rho}-c^{\rho} \partial_{\rho} \tilde{g}^{\gamma \delta}\right] } \\
& +2\left[\frac { 1 } { 4 } c ^ { \mu } \tilde { g } ^ { \lambda \sigma } \left\{\partial_{\mu} b_{\nu}+\partial_{\nu} b_{\mu}+\right.\right. \\
& i\left(\partial_{\mu} \bar{c}_{\lambda}\right)\left(\partial_{\nu} c^{\lambda}\right)+i\left(\partial_{\nu} \bar{c}_{\lambda}\right)\left(\partial_{\mu} c^{\lambda}\right) \\
& \left.+i \partial_{\nu}\left[\left(\partial_{\lambda} \bar{c}_{\mu}\right) c^{\lambda}\right]+i \partial_{\mu}\left[\left(\partial_{\lambda} \bar{c}_{\nu}\right) c^{\lambda}\right]\right\} \\
& +\left[\left(\partial_{\rho} c^{\mu}\right) g^{\rho \lambda}+\left(\partial_{\rho} c^{\lambda}\right) g^{\mu \rho}-c^{\rho} \partial_{\rho} g^{\mu \lambda}\right] \partial_{\lambda}(\sqrt{-g}) \\
& +g^{\mu \lambda} \partial_{\lambda}\left[-\partial_{\rho}\left(c^{\rho} \sqrt{-g}\right)\right] \\
& +\left[\left(\partial_{\sigma} c^{\lambda}\right) \tilde{g}^{\sigma \rho}+\left(\partial_{\sigma} c^{\rho}\right) \tilde{g}^{\lambda \sigma}-c^{\sigma} \partial_{\sigma} \tilde{g}^{\lambda \rho}\right] \Gamma_{\lambda \rho}^{\mu} \\
& \left.-\frac{1}{2} \tilde{g}^{\lambda \rho} g^{\mu \tau}\left(\partial_{\tau} \partial_{\rho} c^{\sigma} g_{\sigma \lambda}+\partial_{\tau} \partial_{\lambda} c^{\sigma} g_{\sigma \rho}+c^{\sigma} \partial_{\tau} \partial_{\sigma} g_{\lambda \rho}\right)\right] .
\end{aligned}
$$

The BRST charge is

$$
Q_{B}=\int d^{3} x J_{B}^{0}
$$

The Faddeev-Popov Lagrangian $\mathcal{L}_{F P}$ is invariant under the transformations $c^{\lambda} \rightarrow e^{-a} c^{\lambda}$ and $\bar{c}_{\rho} \rightarrow e^{a} \bar{c}_{\rho}$. Setting $\delta c^{\lambda}=-c^{\lambda}$ and $\delta \bar{c}_{\lambda}=\bar{c}_{\lambda}$,

$$
\begin{aligned}
& \frac{\partial \mathcal{L}_{F P}}{\partial\left(\partial_{\mu} c^{\lambda}\right)} \delta_{\text {conf }}\left(c^{\lambda}\right)+\frac{\partial \mathcal{L}_{F P}}{\partial\left(\partial_{\mu} \bar{c}_{\lambda}\right)} \delta\left(\bar{c}_{\lambda}\right)= \\
& -\frac{i}{\tilde{\kappa}^{2}}\left[\left(\partial_{\lambda} \bar{c}_{\nu}\right) \tilde{g}^{\mu \nu}+\left(\partial_{\sigma} \bar{c}_{\lambda}\right) \tilde{g}^{\sigma \mu}\right] c^{\lambda} \\
& \quad-\frac{i}{\tilde{\kappa}^{2}}\left[\left(\partial_{\sigma} c^{\mu}\right) \tilde{g}^{\sigma \lambda}+\left(\partial_{\sigma} c^{\lambda}\right) \tilde{g}^{\mu \sigma}-c^{\sigma} \partial_{\sigma} \tilde{g}^{\mu \lambda}\right] \bar{c}^{\lambda} .
\end{aligned}
$$

It equals

$$
\begin{aligned}
& i \tilde{g}^{\mu \lambda}\left[\bar{c}_{\rho}\left(\partial_{\lambda} c^{\rho}\right)-\left(\partial_{\lambda} \bar{c}_{\rho}\right) c^{\rho}\right]+i \alpha b^{\rho} \bar{c}_{\rho} c^{\mu}+\partial_{\lambda} G^{\lambda \mu} \\
& G^{\lambda \mu}=i \bar{c}_{\rho}\left(\tilde{g}^{\lambda \rho} c^{\mu}-\tilde{g}^{\mu \rho} c^{\lambda}\right) .
\end{aligned}
$$

because

$$
\begin{aligned}
& i \tilde{g}^{\mu \lambda}\left[\bar{c}_{\rho}\left(\partial_{\lambda} c^{\rho}\right)-\left(\partial_{\lambda} \bar{c}_{\rho}\right) c^{\rho}\right]+i \alpha b^{\rho} \bar{c}_{\rho} c^{\mu} \\
& \quad+\partial_{\lambda}\left[i \bar{c}_{\rho}\left(\tilde{g}^{\lambda \rho} c^{\mu}-\tilde{g}^{\mu \rho} c^{\lambda}\right)\right] \\
& =i \tilde{g}^{\mu \lambda}\left[\bar{c}_{\rho}\left(\partial_{\lambda} c^{\rho}\right)-\left(\partial_{\lambda} \bar{c}_{\rho}\right) c^{\rho}\right]+i \alpha b^{\rho} \bar{c}_{\rho} c^{\mu} \\
& \quad+i\left(\partial_{\lambda} \bar{c}_{\rho}\right) c^{\mu} \tilde{g}^{\lambda \rho}+i \bar{c}_{\rho}\left(\partial_{\lambda} c^{\mu}\right) \tilde{g}^{\lambda \rho}+i \bar{c}_{\rho}\left(\partial_{\lambda} \tilde{g}^{\lambda \rho}\right) c^{\lambda} \\
& \quad-i\left(\partial_{\lambda} \bar{c}_{\rho}\right) c^{\lambda} \tilde{g}^{\mu \rho}-i \bar{c}_{\rho}\left(\partial_{\lambda} c^{\lambda}\right) \tilde{g}^{\mu \rho}-i \bar{c}_{\rho}\left(\partial_{\lambda} \tilde{g}^{\mu \rho}\right) c^{\lambda}
\end{aligned}
$$

which differs from $\frac{\partial \mathcal{L}_{F P}}{\partial\left(\partial_{\mu} c^{\lambda}\right)} \delta_{\text {conf }}\left(c^{\lambda}\right)+\frac{\partial \mathcal{L}_{F P}}{\partial\left(\partial_{\mu} \bar{c}_{\lambda}\right)} \delta_{\text {conf }}\left(\bar{c}_{\lambda}\right)$ by $i\left(\partial_{\lambda} \bar{c}_{\rho}\right) c^{\mu} \tilde{g}^{\lambda \rho}-i \bar{c}_{\rho}\left(\partial_{\lambda} c^{\lambda}\right) \tilde{g}^{\mu \rho}$, given that $\left[b_{\lambda}, c_{\rho}\right]=\left[b_{\lambda}, \bar{c}_{\rho}\right]=$ $0,\left\{c^{\lambda}, \bar{c}_{\rho}\right\}=0$ and $i \alpha b^{\rho} \bar{c}_{\rho} c^{\mu}+i \bar{c}_{\rho}\left(\partial_{\lambda} \tilde{g}^{\lambda \rho}\right) c^{\mu}=0$. Since $\partial_{\lambda} c^{\lambda}$ and $\partial_{\lambda} \bar{c}^{\lambda}$ are scalars, and $\partial_{\lambda} c^{\lambda} \rightarrow e^{-a} \partial_{\lambda} c^{\lambda}, \partial_{\lambda} \bar{c}^{\lambda} \rightarrow e^{a} \partial_{\lambda} \bar{c}^{\lambda}$ under conformal transformations, both divergences can be set equal to zero.

Removing $\partial_{\mu} G^{\mu \lambda}$ from the current, a conformal current

$$
J_{c}^{\mu}=i \tilde{g}^{\mu \lambda}\left[\bar{c}_{\rho}\left(\partial_{\lambda} c^{\rho}\right)-\left(\partial_{\lambda} \bar{c}_{\rho}\right) c^{\rho}\right]+i \alpha b^{\rho} \bar{c}_{\rho} c^{\mu}
$$

may be defined with a vanishing divergence

$$
\begin{aligned}
\partial_{\mu} J_{c}^{\mu} & =i\left(\partial_{\mu} \tilde{g}^{\mu \lambda}\right)\left[\bar{c}_{\rho}\left(\partial_{\lambda} c^{\rho}\right)-\left(\partial_{\lambda} \bar{c}_{\rho}\right) c^{\rho}\right]+i b^{\rho} \bar{c}_{\rho} c^{\mu} \\
& +i \tilde{g}^{\mu \lambda}\left[\left(\partial_{\mu} \bar{c}_{\rho}\right)\left(\partial_{\lambda} c^{\rho}\right)-\left(\partial_{\mu} \partial_{\lambda} \bar{c}_{\rho}\right) c^{\rho}-\left(\partial_{\lambda} \bar{c}_{\rho}\right) \partial_{\mu} c^{\rho}\right] \\
& +i \alpha \partial_{\mu} b^{\rho} \bar{c}_{\rho} c^{\mu}+i \alpha b^{\rho}\left(\partial_{\mu} \bar{c}_{\rho}\right) c^{\mu}+i \alpha b^{\rho} \bar{c}_{\rho} \partial_{\mu} c^{\mu} \\
& =-i \alpha b^{\lambda}\left[\bar{c}_{\rho}\left(\partial_{\lambda} c^{\rho}\right)-\left(\partial_{\lambda} \bar{c}_{\rho}\right) c^{\rho}\right] \\
& +i \alpha \bar{c}_{\rho}\left[b^{\lambda} \partial_{\lambda} c^{\rho}-b^{\rho}\left(\partial_{\lambda} c^{\rho}\right)-\left(\partial_{\lambda} b^{\rho}\right) c_{\rho}\right] \\
& +i \tilde{g}^{\mu \lambda}\left(\partial \bar{c}_{\rho}\right)\left(\partial_{\lambda} c^{\rho}\right)-i \alpha b^{\lambda}\left[\left(\partial_{\rho} \bar{c}_{\lambda}\right)+\left(\partial_{\lambda} \bar{c}_{\rho}\right)\right] c^{\rho} \\
& -i \tilde{g}^{\mu \lambda}\left(\partial_{\lambda} \bar{c}_{\rho}\right) \partial_{\mu} c^{\rho} \\
& +i \alpha \partial_{\mu} b^{\rho} \bar{c}_{\rho} c^{\mu}+i \alpha b^{\rho}\left(\partial_{\mu} \bar{c}_{\rho}\right) c^{\mu}+i \alpha b^{\rho} \bar{c}_{\rho} \partial_{\mu} c^{\mu} \\
& =0
\end{aligned}
$$

The integral of the conformal current

$$
\begin{array}{r}
Q_{c}=\frac{1}{\tilde{\kappa}^{2}} \int d^{3} x J_{c}^{0}=\frac{1}{\tilde{\kappa}^{2}} \\
\int d^{3} x\left[i \tilde{g}^{0 \lambda}\left[\bar{c}_{\rho}\left(\partial_{\lambda} c^{\rho}\right)-\left(\partial_{\lambda} \bar{c}_{\rho}\right) c^{\rho}\right]+i \alpha b^{\rho} \bar{c}_{\rho} c^{0}\right] .
\end{array}
$$

The commutator $\left[Q_{B}, Q_{c}\right]$ is determined by the BRST variation of the conformal current and the conformal variation of the BRST current. The first variation $\delta_{B R S T} J_{c}^{\mu}$ equals

$$
\begin{aligned}
& \delta_{B R S T}\left[i \tilde{g}^{\mu \lambda}\left[\bar{c}_{\rho}\left(\partial_{\lambda} c^{\rho}\right)-\left(\partial_{\lambda} \bar{c}_{\rho}\right) c^{\rho}\right]+i \alpha b^{\rho} \bar{c}_{\rho} c^{\mu}\right]= \\
& i\left[\left(\partial_{\rho} c^{\mu}\right) \tilde{g}^{\rho \lambda}+\left(\partial_{\rho} c^{\lambda}\right) \tilde{g}^{\mu \rho}-c^{\rho} \partial_{\rho} \tilde{g}^{\mu \lambda}\right] \\
& {\left[\bar{c}_{\sigma}\left(\partial_{\lambda} c^{\sigma}\right)-\left(\partial_{\lambda} \bar{c}_{\sigma}\right) c^{\sigma}\right]} \\
& +i \tilde{g}^{\mu \lambda}\left[i b_{\rho}\left(\partial_{\lambda} c^{\rho}\right)-\bar{c}_{\rho}\left(\partial_{\lambda}\left(c^{\sigma} \partial_{\sigma}\right) c^{\rho}\right)\right. \\
& \left.-i\left(\partial_{\lambda} b_{\rho}\right) c^{\rho}+\left(\partial_{\lambda} \bar{c}_{\rho}\right) c^{\sigma} \partial_{\sigma} c^{\rho}\right] \\
& -\alpha b^{\rho} b_{\rho} c^{\mu}-i \alpha b^{\rho} \bar{c}_{\rho} c^{\lambda} \partial_{\lambda} c^{\mu} .
\end{aligned}
$$


which can be cast in the form

$$
\begin{aligned}
& \left.-\alpha b^{\rho} b_{\rho} c^{\mu}-i \alpha b^{\rho} \bar{c}_{\rho} c^{\lambda} \partial_{\lambda} c^{\mu}-\tilde{g}^{\mu \lambda}\left[b_{\rho}\left(\partial_{\lambda} c^{\rho}\right)-\left(\partial_{\lambda} b_{\rho}\right) c^{\rho}\right)\right] \\
& +i \tilde{g}^{\mu \lambda}\left[\left(\partial_{\rho} \partial_{\lambda} \bar{c}_{\sigma}\right) c^{\rho} c^{\sigma}+\left(\partial_{\rho} \bar{c}_{\sigma}\right) \partial_{\lambda} c^{\rho} c^{\sigma}+2 \partial_{\lambda} \bar{c}_{\sigma} c^{\rho} \partial_{\rho} c^{\sigma}\right] \\
& -i \tilde{g}^{\mu \lambda}\left[\partial_{\lambda}\left(\partial_{\rho} \bar{c}_{\sigma} c^{\rho} c^{\sigma}\right)+\partial_{\lambda}\left(\bar{c}_{\sigma} c^{\rho} \partial_{\rho} c^{\sigma}\right)\right] \\
& +i \partial_{\rho}\left(c^{\mu} \tilde{g}^{\rho \lambda}\left[\bar{c}_{\sigma}\left(\partial_{\lambda} c^{\sigma}\right)-\left(\partial_{\lambda} \bar{c}_{\sigma}\right) c^{\sigma}\right]\right) \\
& +i \partial_{\rho}\left(c^{\lambda} \tilde{g}^{\mu \rho}\left[\bar{c}_{\sigma}\left(\partial_{\lambda} c^{\sigma}\right)-\left(\partial_{\lambda} \bar{c}_{\sigma}\right) c^{\sigma}\right]\right) \\
& -i \partial_{\rho}\left(c^{\rho} \tilde{g}^{\mu \lambda}\left[\bar{c}_{\sigma}\left(\partial_{\lambda} c^{\sigma}\right)-\left(\partial_{\lambda} \bar{c}_{\sigma}\right) c^{\sigma}\right]\right) \\
& +i \alpha c^{\mu}\left[\bar{c}_{\sigma} b^{\sigma}\left(\partial_{\lambda} c^{\lambda}\right)+\bar{c}_{\sigma}\left(\partial_{\lambda} b^{\sigma}\right) c^{\lambda}+b^{\lambda}\left(\partial_{\sigma} \bar{c}_{\lambda}\right) c^{\sigma}\right] \\
& +i \alpha c^{\lambda} b^{\mu}\left[\bar{c}_{\sigma}\left(\partial_{\lambda} c^{\sigma}\right)-\left(\partial_{\lambda} \bar{c}_{\sigma}\right) c^{\sigma}\right] \\
& +i\left(\partial_{\rho} c^{\rho}\right) \tilde{g}^{\mu \lambda}\left[\bar{c}_{\sigma}\left(\partial_{\lambda} c^{\sigma}\right)-\left(\partial_{\lambda} \bar{c}_{\sigma}\right) c^{\sigma}\right]
\end{aligned}
$$

Since

$$
\begin{aligned}
& -i \tilde{g}^{\mu \lambda}\left[\partial_{\lambda}\left(\partial_{\rho} \bar{c}_{\sigma} c^{\rho} c^{\sigma}\right)+\partial_{\lambda}\left(\bar{c}_{\sigma} c^{\rho} \partial_{\rho} c^{\sigma}\right)\right] \\
& =-i \partial_{\lambda}\left(\tilde{g}^{\mu \lambda}\left(\partial_{\rho} \bar{c}_{\sigma} c^{\rho} c^{\sigma}+\bar{c}_{\sigma} c^{\rho} \partial_{\rho} c^{\sigma}\right)\right] \\
& -i \alpha b^{\mu}\left[\partial_{\rho} \bar{c}_{\sigma} c^{\rho} c^{\sigma}+\bar{c}_{\sigma} c^{\rho} \partial_{\rho} c^{\sigma}\right]
\end{aligned}
$$

there is a simplification of the combination

$$
\begin{aligned}
& i \alpha c^{\lambda} b^{\mu}\left[\bar{c}_{\sigma}\left(\partial_{\lambda} c^{\sigma}\right)-\left(\partial_{\lambda} \bar{c}_{\sigma}\right) c^{\sigma}\right] \\
& -i \alpha b^{\mu}\left[\partial_{\rho} \bar{c}_{\sigma} c^{\rho} c^{\sigma}+\bar{c}_{\sigma} c^{\rho} \partial_{\rho} c^{\sigma}\right]=-2 i \alpha b^{\mu} \bar{c}_{\sigma} c^{\rho} \partial_{\rho} c^{\sigma}
\end{aligned}
$$

and a cancellation of terms in the first and sixth lines

$$
\begin{aligned}
& i \alpha c^{\mu} \bar{c}_{\sigma} \partial_{\lambda} b^{\sigma} c^{\lambda}+i \alpha c^{\mu} \bar{c}_{\sigma} b^{\sigma} \partial_{\lambda} c^{\lambda}+i \alpha c^{\mu} b^{\lambda} \partial_{\sigma} \bar{c}_{\lambda} c^{\sigma} \\
& \quad-i \alpha b^{\rho} \bar{c}_{\rho} c^{\lambda} \partial_{\lambda} c^{\mu} \\
& \quad=i \alpha \partial_{\lambda}\left(c^{\mu} \bar{c}_{\sigma} b^{\sigma} c^{\lambda}\right)-i \alpha \partial_{\lambda} c^{\mu} \bar{c}_{\sigma} b^{\sigma} c^{\lambda}-i \alpha b^{\rho} \bar{c}_{\rho} c^{\lambda} \partial_{\lambda} c^{\mu} \\
& \quad=i \alpha \partial_{\lambda}\left(c^{\mu} \bar{c}_{\sigma} b^{\rho} c^{\lambda}\right)
\end{aligned}
$$

yielding

$$
\begin{aligned}
-\alpha b^{\rho} b_{\rho} c^{\mu}-\tilde{g}^{\mu \lambda}\left[b_{\rho}\left(\partial_{\lambda} c^{\rho}\right)-\left(\partial_{\lambda} b_{\rho}\right) c^{\rho}\right] & (81) \\
& +i \tilde{g}^{\mu \lambda}\left[\left(\partial_{\rho} \partial_{\lambda} \bar{c}_{\sigma}\right) c^{\rho} c^{\sigma}+\left(\partial_{\rho} \bar{c}_{\sigma}\right) \partial_{\lambda} c^{\rho} c^{\sigma}+2 \partial_{\lambda} \bar{c}_{\sigma} c^{\rho} \partial_{\rho} c^{\sigma}\right] \\
& -2 i \alpha b^{\mu} \bar{c}_{\sigma} c^{\rho} \partial_{\rho} c^{\sigma}+i\left(\partial_{\rho} c^{\rho}\right) \tilde{g}^{\mu \lambda}\left[\bar{c}_{\sigma}\left(\partial_{\lambda} c^{\sigma}\right)-\left(\partial_{\lambda} \bar{c}_{\sigma}\right) c^{\sigma}\right] \\
& + \text { total derivative terms }
\end{aligned}
$$

for $\delta_{B R S T} J_{c}^{\mu}$. The value of $\alpha$ is a gauge choice. Setting $\alpha=0$, integration by parts with Eq.(3.24) gives

$$
\begin{aligned}
& 2 \tilde{g}^{\mu \lambda}\left(\partial_{\lambda} b_{\rho}\right) c^{\rho}+i \tilde{g}^{\mu \lambda}\left[\left(\partial_{\lambda} \bar{c}_{\sigma}\right)\left(c^{\rho} \partial_{\rho} c^{\sigma}-\partial_{\rho} c^{\rho} c^{\sigma}\right)\right. \\
& \left.+\partial_{\rho} \bar{c}_{\sigma} \partial_{\lambda} c^{\rho} c^{\sigma}\right] \\
& +i \tilde{g}^{\mu \lambda}\left[-\bar{c}_{\sigma} \partial_{\lambda} c^{\sigma}\left(\partial_{\rho} c^{\rho}\right)+\left(\partial_{\lambda} \bar{c}_{\sigma}\right)\left(\partial_{\rho} c^{\rho}\right) c^{\sigma}\right] \\
& + \text { total derivative terms } \\
& =2 \tilde{g}^{\mu \lambda}\left(\partial_{\lambda} b_{\rho}\right) c^{\rho}+i \tilde{g}^{\mu \lambda}\left[\left(\partial_{\lambda} \bar{c}_{\sigma}\right)\left(c^{\rho} \partial_{\rho} c^{\sigma}-\partial_{\rho} c^{\rho} c^{\sigma}\right)\right. \\
& \left.-\bar{c}_{\sigma} \partial_{\lambda} c^{\sigma}\left(\partial_{\rho} c^{\rho}\right)\right] \\
& + \text { total derivative terms }
\end{aligned}
$$

By Eq.(4.18), integration by parts and the equation

$$
\tilde{g}^{\mu \nu} \partial_{\mu} \partial_{\nu} c^{\rho}=\alpha\left[b^{\lambda}\left(\partial_{\mu} c^{\rho}\right)-b^{\rho}\left(\partial_{\lambda} c^{\lambda}\right)-\left(\partial_{\lambda} b^{\rho}\right) c^{\lambda}\right]
$$

which may be set equal to zero when $\alpha=0, \delta_{B R S T} J_{c}^{\mu}$ equals the sum of a curvature term $4 R^{\mu}{ }_{\rho} c^{\rho}$ and total derivatives. Regarding the spin-2 fields as perturbations about the background metric, $R_{\mu \nu}$ can be set equal to zero either on flat space or through the vacuum gravitational field equations. Similarly, it may be deduced from Eq.(4.21) that $\delta_{c} J_{B}^{\mu}=-J_{B}^{\mu}$. Then $\delta_{B R S T} J_{c}^{\mu}-\delta_{c} J_{B R S T}^{\mu}$ can be equated to the sum of $-J_{B}^{\mu}$ and total derivative terms [10]. The projection of $\left[Q_{B}, Q_{c}\right]$ onto two dimensions may be identified with $-Q_{B}$. Therefore, the action of the commutator will vanish on physical states satisfying $Q_{B}|\phi\rangle=0$.

The ghost fields therefore provide a method for incorporating scale invariance into the gravitational theory. It may be noted that the variation $\delta_{\text {conf }}\left(\tilde{g}^{\mu \nu}\right)$ has been set equal to zero. It vanishes most naturally in two dimensions because, $g_{\mu \nu} \rightarrow e^{a} g_{\mu \nu}, \sqrt{-g} \rightarrow \sqrt{e^{2 a}(-g)}=e^{a} \sqrt{-g}$, and $\tilde{g}^{\mu \nu}=g^{\mu \nu} \sqrt{-g} \rightarrow \tilde{g}^{\mu \nu}$. These fields can be projected onto two-dimensional string worldsheets to provide the content of a theory invariant under scale transformations. The projection of the Faddeev-Popov action to two dimensions is

$$
\begin{aligned}
& \left.\mathcal{L}_{F P}\right|_{\Sigma}=-\frac{1}{\tilde{\kappa}^{2}}\left[\left(\partial_{\alpha} b_{\beta}\right) \tilde{h}^{\alpha \beta}+i\left(\partial_{\alpha} \bar{c}_{\beta}\right)\left(\left(\partial_{\gamma} c^{\alpha}\right) \tilde{h}^{\gamma \beta}\right.\right. \\
& \left.\left.+\left(\partial_{\gamma} c^{\beta}\right) \tilde{h}^{\gamma \alpha}-c^{\gamma} \partial_{\gamma} \tilde{h}^{\alpha \beta}\right)-\frac{\alpha_{2}}{2} \delta^{\alpha \beta} b_{\alpha} b_{\beta}\right]
\end{aligned}
$$

where $\tilde{h}^{\alpha \beta}=h^{\alpha \beta} \sqrt{h}$, with $h_{\alpha \beta}$ being the two-dimensional metric, which differs from the free-fermion $b c$ theory on a Riemann surface arising from the gauge fixing of the string action. It is closer to the action for $W_{3}$ gravity in the conformal gauge can be reformulated in the higher derivative gauge $[11]$ to be

$$
\begin{aligned}
& I_{W_{3}}=\int d^{2} z\left[-\frac{1}{2} \bar{\partial} \varphi^{\alpha} \partial \varphi_{\alpha}\right. \\
& \left.-h T_{m a t}+\pi_{h} \bar{\partial} h-b \bar{\partial}(\bar{\partial} c+c \partial h+\partial c h),\right]
\end{aligned}
$$

where $h$ is an unfixed component of the worldsheet metric $h_{\alpha \beta}=\left(\begin{array}{ll}1 & 0 \\ 0 & h\end{array}\right)$ equal to the determinant, $b$ and $c$ are ghost fields, $\pi_{h}$ is an auxiliary field and $T_{m a t}$ is the matter energy-momentum tensor, after a FFBRST transformation of the scalars $\varphi^{i}$, the ghost fields, $h$ and $\pi_{h}$ [12]. The ghost terms in the Lagrangian now consist of two derivatives, which occur also in the projection of the four-dimensional gravitactional action to the worldsheet.

The gravitational interaction between two masses was described initially by a force law which has the same form as that of electrostatics, except that masses must have the same sign. The gravitational potential satisfies the Poisson equation $\nabla^{2} \varphi=-8 \pi G \rho$, where $\rho$ is the mass density, which may be derived in a weak-field limit of general relativity. Without the source term, in the de Donder gauge, the equation for the spin-two excitation is $\square h_{\mu \nu}=0$. Since $\left.h_{00}=-2 \varphi, h_{i j}=-2 \phi \delta_{[} i j\right], \square \varphi=0$. The Green function for the d'Alembertian in four dimensions is $\frac{1}{\left|x^{\mu} x_{\mu}\right|}$. By a Fourier transform, the propagator can be represented in momentum space as $\frac{1}{p^{2}}$. Therefore, the model may be quantized similarly to electrodynamics, with the vector fields replaced by scalar fields. To incorporate the effects of mass by analogy with the Poisson's law, with 
the gravitational potential of a mass $\varphi=-G \frac{m}{r}$ and $\left.-4 \pi G \rho=-4 \pi G \frac{m}{\frac{4}{3} \pi r^{3}}=-3 G \frac{m}{r^{3}}=\frac{3}{[} r^{2}\right] \varphi$, consider the equation $\left(\square-\frac{3}{r^{2}}\right) \varphi=0$. The Green function for this differential operator would be $\frac{(m r)^{\sqrt{3}}}{\left|x^{\mu} x_{\mu}\right|}$. The radial form is $\frac{(m r)^{\sqrt{3}}}{r^{2}}$.

Removing the field strength term from scalar electrodynamics, and replacing the gauge potential coupling with a weak-field connection term, the Lagrangian would have the form

$$
\begin{aligned}
\mathcal{L}_{\text {scalar gravity }} & =\frac{1}{2} D^{\mu} \varphi^{\dagger} D_{\mu} \varphi+\frac{1}{2} m^{2} \varphi^{\dagger} \varphi \\
D_{\mu} \varphi & =\left(\partial_{\mu}+\Gamma_{\mu}\right) \varphi
\end{aligned}
$$

Even though the connection is not a tensor field, spatial components of the Christoffel symbol are given by a gradient of the potential and $\Gamma_{\mu}$ may be set equal to $\partial_{\alpha} \varphi$. Then

$$
\mathcal{L}_{\text {scalar gravity }}=\frac{1}{2}\left(1+\varphi^{\dagger}\right)(1+\varphi) \partial^{\mu} \varphi^{\dagger} \partial_{\mu} \varphi+\frac{1}{2} m^{2} \varphi^{\dagger} \phi .
$$

Let $m=0$ and $\tilde{\varphi}=1+\varphi$. The Lagrangian then equals

$$
\mathcal{L}_{\text {scalar gravity }}(m=0)=\tilde{\varphi}^{\dagger} \tilde{\varphi} \partial^{\mu} \varphi \dagger \partial_{\mu} \tilde{\varphi}
$$

Under the transformations $\tilde{\phi} \rightarrow e^{i \alpha(x)} \tilde{\varphi}(x)$ and $\tilde{\varphi}^{\dagger} \rightarrow$ $e^{-i \alpha(x)} \tilde{\varphi}^{\dagger}(x)$,

$$
\begin{aligned}
& \delta \mathcal{L}_{\text {scalar gravity }}(m=0)=\tilde{\phi}^{\dagger} \tilde{\varphi}\left\{\partial^{\mu} \tilde{\varphi}^{\dagger} \partial_{\mu} \tilde{\varphi}+\partial^{\mu} \alpha \partial_{\mu} \varphi \varphi^{\dagger} \varphi\right. \\
& \left.-i \partial^{\mu} \alpha \tilde{\varphi}^{\dagger} \partial_{\mu} \tilde{\varphi}+i \partial_{\mu} \alpha \tilde{\varphi} \partial^{\mu} \tilde{\varphi}^{\dagger}\right\} \\
& -\tilde{\phi}^{\dagger} \tilde{\varphi} \partial^{\mu} \tilde{\varphi}^{\dagger} \partial_{\mu} \tilde{\varphi} \\
& =\left(\tilde{\varphi}^{\dagger} \tilde{\varphi}\right) \partial^{\mu} \alpha \partial_{\mu} \alpha+i \partial_{\mu}\left\{\tilde{\varphi}^{\dagger} \tilde{\varphi} \alpha\left[\tilde{\varphi} \partial^{\mu} \tilde{\varphi}^{\dagger}-\tilde{\varphi}^{\dagger} \partial^{\mu} \tilde{\varphi}\right\}\right. \\
& -i \alpha\left[\left(\tilde{\varphi} \partial^{\mu} \tilde{\varphi}^{\dagger}\right)^{2}-\left(\tilde{\varphi}^{\dagger} \partial^{\mu} \tilde{\varphi}\right)^{2}\right] .
\end{aligned}
$$

Since $\partial_{\mu}\left(\tilde{\varphi} \partial^{\mu} \tilde{\varphi}^{\dagger}\right)=\partial_{\mu}\left(\tilde{\varphi}^{\dagger} \partial^{\mu} \tilde{\varphi}\right), \tilde{\varphi} \partial^{\mu} \tilde{\varphi}^{\dagger}=\tilde{\varphi}^{\dagger} \partial^{\mu} \tilde{\varphi}+k^{\mu}$, where $\partial_{\mu} k^{\mu}=0$ and

$-i \alpha\left[\left(\tilde{\varphi} \partial^{\mu} \tilde{\varphi}^{\dagger}\right)^{2}-\left(\tilde{\varphi}^{\dagger} \partial^{\mu} \tilde{\varphi}\right)^{2}\right]=-i \alpha \partial_{\mu}\left(\tilde{\varphi}^{\dagger} \tilde{\varphi}\right) k^{\mu}=$ $-i \alpha \partial_{\mu}\left(\tilde{\varphi}^{\dagger} \tilde{\varphi} k^{\mu}\right)$, which is a total derivative if $\partial_{\mu} \alpha=0$, $k^{\mu}=0$ or $\partial_{\mu} \alpha\left(\tilde{\phi}^{\dagger} \tilde{\phi} k^{\mu}\right)=0$. The condition $\tilde{\varphi} \partial^{\mu} \tilde{\varphi}^{\dagger}=\tilde{\varphi}^{\dagger} \partial^{\mu} \tilde{\varphi}$ requires $\tilde{\varphi}$ to have a constant phase, and therefore it cannot be preserved under local gauge transformations with $\partial_{\mu} \alpha \neq 0$.

While ghosts may be eliminated in this model through a decoupling from the scalar field, the gauge may be fixed, reducing the symmetry from a local to global $\mathrm{U}(1)$ invariance, by adding the Lagrange multiplier term $\frac{1}{2} \int d^{4} x c_{\mu}\left(\tilde{\varphi} \partial^{\mu} \tilde{\varphi}^{\dagger}\right.$

$\left.-\tilde{\varphi}^{\dagger} \partial^{\mu} \tilde{\varphi}\right)$. The projection to two dimensions is $\frac{1}{2} \int d^{2} z c(\tilde{\varphi} \partial \overline{\tilde{\varphi}}-\overline{\tilde{\varphi}} \partial \tilde{\varphi})$. The sum of the projected ghost action and gauge-fixing term can be projected onto two-dimensional string worldsheet

$$
\frac{1}{2} \int[a \bar{\partial} b+b \bar{\partial} c+c(\tilde{\varphi} \partial \overline{\tilde{\varphi}}-\overline{\tilde{\varphi}} \partial \tilde{\phi})]
$$

The Euler-Lagrange equation for $c$ is

$$
\bar{\partial} b=\tilde{\varphi} \partial \overline{\tilde{\varphi}}-\overline{\tilde{\varphi}} \partial \tilde{\varphi}
$$

and variation with respect to $a$ gives

$$
\bar{\partial} b=0
$$

which fixes the gauge. Variation with respect to $b$ yields $\bar{\partial} c=0$. The two relations also may derived from the standard $b c$ action $\frac{1}{2} \int b \bar{\partial} c$.

It has been suggested that all of the supergravity amplitudes at tree level can be given by a universal formula which is valid for a class of $N^{k} M H V$ as well as $M H V$ amplitudes [13]. This conclusion is based, however, on a single trace decomposition of the amplitude which follows only for rational curves in twistor space of degrees $d_{1}$ and $d_{2}$ with one intersection. The complex dimension is one for each curve, yielding complex codimension $c_{i}$, $i=1$, 2, equal to two, and $\sum_{i=1}^{2} c_{i}=4$, which is larger than that of the twistor space, and therefore, the upper bound $d_{1} d_{2}$ is not relevant. Nevertheless, there may exist multiple intersections of the curves and the number can be counted through Floer theory [14]. The $N^{k} M H V$ amplitudes are known to introduce higher-genus curves, which require conformal supergravity and quartic poles in the factorization of the amplitudes [15]. The ghost structure [16] and the divergences [17] in conformal supergravity have been described. The equations of the bosonic sector of supergravity theory coupled to scalar and antisymmetric tensor fields have been derived from the vanishing of anomalies in operator products in worldsheet gravity [20]. The ambitwistor string can be formulated in four and ten dimensions [18], and a gravitational action, summed in a perturbation series, may be derived from the twistor string amplitudes [19].

The Liouville action describing the dynamics of a scalar mode in string theory may be generalized to the Donaldson action with a variation that gives the equation for hermitian structures in holomorphic vector bundles both on two-dimensional surfaces and four-manifolds [21]. Therefore, both the dependence of the metric on the scale and extra embedding degrees of freedom in the tangent and normal bundles on the three-dimensional geometries foliating the four-manifold may be investigated with this action.

The BRST symmetry can be extended to a quadratic gravity action, renormalizable in the generalized sense [22], with couplings to a scalar field in a unitary theory. The scale invariance of the Faddeev-Popov action may be projected to this symmetry in two dimensions, which then yields a quantum theory with conformal invariance. The existence of a conformal invariance of a theory with scale invariance in four dimensions also follows perturbatively given a set of conditions including unitarity, Poincare invariance in the background space-time, a discrete spectrum in the scaling dimension and the existence of a scale current [23]. The physical states invariant under BRST transformations in four dimensions will be projected to states for which the action of the BRST and conformal transformations commute in in two dimensions. The BRST invariance of the induced two-dimensional action then 
would be valid on any Riemann surface with an ideal boundary of zero linear measure.

\section{Conclusion}

The BRST transformations of terms in the induced supergravity action have been extended to give to superspace to establish whether the invariance is preserved at higher genus. It is found that three extra sets of terms arise in the transformation. Two of the terms can be integrated to give zero on a compact surface and do not affect the induced action. The third term is an integral of an expression that includes an anticommutator of a superspace derivative and the BRST transformation that vanishes. The action of the BRST operator on the second anomaly term $\mathcal{A}_{2}$ therefore yields an expression consisting of differentials, with the sum of $T_{\lambda} d \lambda$ and $\bar{T}_{\bar{\lambda}} d \bar{\lambda}$, proving the BRST invariance of the anomaly through one of the superspace techniques [4]. By contrast, a direct evaluation of the BRST transformation of the Wess-Zumino-Polyakov action gives the standard diffeomorphism anomaly, together with several other terms. Since the BRST operator $s$ is required to satisfy $s^{2}=0$, it will be necessary to check the other terms in the variation of $\Gamma_{W Z P}$ in superspace.

The preservation of the anomaly under the action of $s$ is valid in gauge theories where the two transformations do commute. The effective action in two-dimcensional conformal field theories is defined such that the BRST variation yields the anomaly with a total derivative. However, since the domain of the integral is a region of the complex plane exterior to $2 g$ disks at genus $g$, and the integral of any differential would equal a sum of contour integrals over the boundaries of the disks. By $P S L(2 ; \mathbb{C})$ invariance, there are pairwise cancellations of the contour integrals with opposite orientations. A second BRST transformation again will yield derivative terms which generate contour integrals over the curves that occur after BRST transformations of the circular boundaries. The generalization to the superstring action will induce a supergravity action with fermion fields. In addition to diffeomorphism invariance, the st This result would even be verified for the induced action for the bosonic string for a class of infinite-genus surfaces. The contribution of boundaries of surfaces also may be evaluated. It is found that the integrals over the ideal boundaries of infinite-genus surfaces will not yield any additional terms when the harmonic measure is equal to zero.

The conformal current for the Faddeev-Popov action has a vanishing divergence and the commutator of the BRST charge with the conformal charge $Q_{c}$ is nonvanishing. This calculation may be extended to the quadratic gravity action. The Faddeev-Popov ghosts in the quantization of the quadratic gravity action must be projected to ghosts in the quantization of the induced supergravity action of the superstring. On the vacuum state, the action of the commutator would be be projected to zero in two dimensions. The consistency of the projection from four to two dimensions will be required for the embedding of the string dynamics in four dimensions.

The Faddeev-Popov action for general relativity is quadratic in derivatives which will occur in a projection to a two-dimensional surface. Therefore, it is conjectured that a scalar form of the theory of gravity may be developed. A consideration of the weak-field approximation of the gravitational field equations and a Lagrangian by analogy with scalar electrodynamics without the field strength term results an action with complex scalar fields. The gauge invariance is initially a global U(1) symmetry. However, allowing the phase to be position-dependent, equations for $\alpha$ are derived for the variation of the Lagrangian to be a total derivative. Fixing a gauge, the local U(1) invariance is reduced to the global U(1) group, and the ghost action is found. The field equations are found to coincide with that of the ghost action of bosonic string theory and the gauge fixing condition. Therefore, a scalar form of the gravity theory is necessary to establish a relation between the models in two and four dimensions. The extension of the symmetries on the string worldsheet to a complete description of the dynamics in the embedding space may require the inclusion of conformal gravity, which represents the effective theory of the twistor string.

The Weyl invariance of the Faddeev-Popov Lagrangian confirms the characterization of the gravitational action as an effective theory derived from a string sigma model on the Riemann surface. In bosonic string and superstring theory, the equations for the metric to preserve the conformal symmetry at the quantum level are derived from an effective action in a critical dimensions equal to twenty-six and ten respectively. There are conditions also on the metric that arise from variations for a Lagrangian expanded in a series with higher-order curvature combinations. The solutions to these equations to all orders in the sigma model coupling include flat space and several other classes of geometries, which can be compactified over extra coordinates to four dimensions. The scale invariance is recovered only in the ghost sector of gravity, which is consistent with the absence of this symmetry at sufficiently large distances.

\section{References}

[1] F. Delduc and F. Gieres, Anomalies and Effective Actions in TwoDimensional Superspace, Int. J. Mod. Phys. A7 (1992) 1685-1704.

[2] R. Zucchini, A Polylakov Action on Riemann Surfaces, Phys. Lett. 260B (1991) 296-302.

[3] R. Zucchini, A Polyakov Action on Riemann Surfaces (II), Commun. Math. Phys. 152 (1993) 269-297.

[4] J.-P. Ader and H. Kachkachi, Induced Polyakov Supergravity on Riemann Surfaces of Higher Genus, Class. Quant. Grav. 11 (1994) 767-784.

[5] W. Kummer, Gauge-Independence of Anomalies, in:Fields and Particles, eds. H. Mitter and W. Scwheiger, Springer-Verlag, Heidelberg, 1990, pp. 231-254.

[6] F. Delduc and F. Gieres, Beltrami Differentials, Conformal Models and their Supersymmetric Generalizations, Class. Quantum Grav. 7 (1990) 1907-1952. 
[7] I. N. Bernshtein and D. A. Leites, Integral Forms and the Stokes formula on Supermanifolds, Functional Analysis and Its Applications 11 (1977) 45-47.

[8] M. Schaden and D. Zwanziger, Living with Spontaneously Broken BRST Symmetry II. Poincare, Phys. Rev. D92 (2015) 025002:114.

[9] L. Sario and M. Nakai, Classification Theory of Riemann Surfaces, Die Grundlehren der mathematischen Wissenschaften in Einzeldarstellungen mit besondere Berücksichtigun der Anwendungsgebiete, Vol. 164, Springer-Verlag, Heidelberg, 1970.

[10] K. S. Stelle, Renormalization of Higher-Derivative Quantum Gravity, Phys. Rev. D16 (1977) 953-969.

[11] R. Moyahee, C. N. Pope, K. S. Stelle and K.-W. Xu, Nucl. Phys. B433 (1995) 712.

[12] S. Upadhyay, The Conformal Gauge to the Derivative Gauge for Worldsheet Gravity, Phys. Lett. B740 (2015) 341-344.

[13] F. Cachazo and D. Skinner, Gravity from Rational Curves, Phys. Rev. Lett. 110 (2013) 161301:1-4.

[14] A. Floer, Morse Theory for Lagrangian Intersections, J. Diff. Geom. 28 (1988) 513-547.

[15] N. Berkovits and E. Witten, Conformal Supergravity in Twistor Space, J. High Energy Phys. 0804 (2004) 009:1-37.

[16] S. Ferrara and B. Zumino, Structure of Conformal Supergravity, Nucl. Phys. 134B (1978) 301-326.

[17] A. A. Tseytlin, On Divergences in Non-Minimal $N=4$ Conformal Supergravity, J. Phys. A: Math. and Theor. 50 (2017) 48LT01.

[18] Y. Geyer and L. Mason, Supersymmetric S-Matrices from the Worldsheet in 10 \& 11d, Phys. Lett. 804B (2020) 135361: 1-7.

[19] Y. Geyer, Ambitwistor Strings: Worldsheet Approaches to Perturbative Quantum Field Theory, Oxford University D. Phil. Thesis, 2016.

[20] T. Adamo, E. Casali and D. Skinner, A Worldsheet Theory for Supergravity, J. High Energy Phys. 0215 (2015) 116:1-17.

[21] R. Zucchini, Deformation Theory of Holomorphic Vector Bundles, Extended Conformal Symmetry and Extensions of 2D Gravity, Class. Quantum Grav. 11 (1994) 1697-1724.

[22] E. Elizalde, A. G. Jacksanaev, S. D. Odintsov and I. L. Shapiro, A Four-Dimensional Theory for Quantum Gravity with Conformal and Nonconformal Explicit Solutions, Class. Quantum Grav. 12 (1995) 1385-1400.

[23] Y. Nakayama, Scale Invariance vs. Conformal Invariance, Phys. Rep. C569 (2015) 1-93.

Dr Simon Davis received the $\mathrm{PhD}$ degree in Theoretical Physics at the University of Cambridge. His research interests include quantum and and gravitational theory. Elementary particle physics and the unification of the fundamental interactions have been the focus of scientific work continued at Harvard University, the International Centre for Theoretical Physics and the University of Sydney. Investigations in the mathematical fields of geometry and analysis have been continued at the Research Foundation of Southern California. 